\title{
Hydrogel-Hydroxyapatite-Monomeric Collagen Type- I Scaffold with Low-Frequency Electromagnetic field Treatment Enhances Osteochondral Repair in Rabbits
}

\section{Jiyuan Yan}

Tongji Hospital of Tongji Medical College of Huazhong University of Science and Technology

Chaoxu Liu

Tongji Hospital of Tongji Medical College of Huazhong University of Science and Technology

Yingchi Zhang

Tongji Hospital of Tongji Medical College of Huazhong University of Science and Technology

Chang Tu

Wuhan University Renmin Hospital

\section{Ruizhuo Zhang}

Tongji Hospital of Tongji Medical College of Huazhong University of Science and Technology

\section{Xiangyu Tang}

Tongji Hospital of Tongji Medical College of Huazhong University of Science and Technology Department of Radiology

\section{Hao Li}

Tongji Hospital of Tongji Medical College of Huazhong University of Science and Technology

\section{Huaixi Wang}

Henan Provincial People's Hospital

\section{Yongzhuang Ma}

Shanxi Bethune Hospital

Hua Wu ( $\nabla$ wuhua@hust.edu.cn )

Tongji Hospital of Tongji Medical College of Huazhong University of Science and Technology https://orcid.org/0000-0003-1802-1363

\section{Gaohong Sheng}

Tongji Hospital of Tongji Medical College of Huazhong University of Science and Technology

\section{Research Article}

Keywords: Hydrogel, Hydroxyapatite, Monomeric Collagen type I (Col1), Electromagnetic fields, Osteochondral defects, Mesenchymal stem cells 
Posted Date: June 22nd, 2021

DOl: https://doi.org/10.21203/rs.3.rs-632090/v1

License: (9) This work is licensed under a Creative Commons Attribution 4.0 International License. Read Full License

Version of Record: A version of this preprint was published at Stem Cell Research \& Therapy on November 13th, 2021. See the published version at https://doi.org/10.1186/s13287-021-02638-6. 


\section{Abstract}

Background: Cartilage damage is a common medical issue in clinical practice. Complete cartilage repair remains a significant challenge owing to the inferior quality of regenerative tissue.

Methods: In this study, a composite scaffold made of Hydroxyapatite-Collagen type-I (HAC) and PLGAPEG-PLGA thermogel was produced to match the cartilage and subchondral layers in osteochondral defects, respectively. Bone marrow mesenchymal stem cells (BMSC) encapsulated in the thermogel were stimulated by an electromagnetic field (EMF).

Results: The scaffold was evaluated in rabbits, and biological characteristic of BMSCs were measured. Results showed that the scaffold with EMF enhances the repair of osteochondral defects in rabbits, and, in particular, cartilage repair. EMF could promote proliferation and chondrogenic differentiation of BMSCs partly by activating the PI3K/AKT/mTOR and Wnt1/LRP6/ $\beta$-catenin pathway.

Conclusion: Hydrogel-Hydroxyapatite-Monomeric Collagen type-I scaffold with low-frequency EMF treatment has the potential to enhance osteochondral repair.

\section{Introduction}

Articular cartilage is mainly composed of chondrocytes and the extracellular matrix (ECM), such as aggrecan and collagen type 2 (Col2) with complex hierarchical structure [1]. During daily activities, cartilage plays an important role in the transmission of joint loads and lubrication of articular movement [2]. Cartilage damage is a common medical issue caused by trauma, aging, and disorders of cartilage itself and subchondral bone (including osteoarthritis, osteonecrosis, and osteochondritis dissecans) [3, 4]. The chronic pain and dysfunction of affected joints significantly diminish the patient's life quality. Owing to the avascularity of cartilage and low metabolic activity of chondrocytes, such cartilage defects have limited capacity to heal spontaneously $[5,6]$. Several therapeutic strategies have been developed to treat cartilage damage, including bone marrow stimulation (such as microfracture and subchondral bone drilling) [7, 8], autologous chondrocyte implantation [9-11] and autografts transplantation[12, 13]. However, the complete repair of hyaline cartilage remains a major challenge in clinical practice owing to the inferior quality of regenerative tissue $[14,15]$. Moreover, the results of long-term follow-up have revealed a poor clinical prognosis in patients with cartilage defects because of cartilage degradation, regardless of the treatment therapy [16-19].

Recently, tissue engineering has provided novel insights into the treatment of osteochondral defects [20, 21]. Among various biomaterials, injectable thermogels have received significant attention owing to several unique advantages, including minimally invasive implantation, sterilization by filtration, no need for organic solvents, and good plasticity for irregularly shaped defects or cavities [22]. Generally, drugs or cells are easily mixed and encapsulated in aqueous solution (sol state) at ambient temperature. Then, the solution undergoes sol-to-gel transition to form gels when heated. This in situ thermogelling system has been widely applied, for example, in drug delivery, prevention of postoperative adhesion, transcatheter 
arterial embolization, and tissue repair [22-24]. For instance, thermosensitive hydrogel based on poly(lactide-co-glycolide)-poly(ethylene glycol)-poly(lactide-co-glycolide) (PLGA-PEG-PLGA) triblock copolymer are extensively applied in clinical use owing to its injectability, excellent biocompatibility, and biodegradability $[25,26]$. Furthermore, a study indicated that this thermogel performed better than fibrin gel with regard to relieving symptoms of rheumatoid arthritis (RA) using BMSCs culture [27]. In view of cartilage tissue engineering, the PLGA-PEG-PLGA copolymer simulates a 3D network structure, similar to that of native cartilage, to support proliferation and differentiation of BMSCs. Furthermore, the main product of poly(lactide-co-glycolide) (PLGA) degradation is glutamate, which is the most abundant amino acid in articular cartilage [28]. Cell-based tissue engineering is currently a common and effective therapy for cartilage regeneration. Among the numerous cell types, mesenchymal stem cells (MSCs)--BMSCs, in particular--are apparently the most promising candidates owing to their capacity of self-renewal, proliferation, and multi-lineage differentiation. [29, 30].

Remarkably, increasing evidence has demonstrated that physical stimuli such as electromagnetic fields (EMFs) could promote chondrogenic differentiation of BMSCs [31].The EMF exposure induced chondrocyte proliferation and differentiation, and ECM synthesis by variable potential mechanisms, including regulating calcium channel in cell membrane [32, 33], increasing transforming growth factor beta (TGF $\beta$ ) and bone morphogenetic protein 2 (BMP2) expressions [34, 35], and modulating MSCs secretome and paracrine function [36]. Stefani et al. [37] reported that EMF stimulation improved repair and integration of engineered constructs in an animal model. In addition, EMF was suggested to exert an anti-inflammatory effect through improving immunomodulatory function of MSCs [38, 39]. Notably, another advantage was that EMF could significantly expedite osteogenesis of MSCs and bone regeneration $[40,41]$. The reconstruction of subchondral bone is critical to cartilage restoration, as cartilage defects frequently involve destruction of the subchondral bone. Thus, EMF has attracted considerable attention for repairing osteochondral defects with engineered constructs.

In this study, we explored the effect of sinusoidal EMF (SEMF) on osteochondral repair combined with BMSCs-based tissue engineering, especially cartilage regeneration, using the PLGA-PEG-PLGA thermogel, and its underlying molecular mechanisms. According to our previous study, the parameters of EMF (15 $\mathrm{Hz}, 1 \mathrm{mT}, 4 \mathrm{~h} /$ day) could efficiently facilitate proliferation and chondrogenic differentiation of BMSCs in vitro [42]. After exposure to EMF for 7 and 14 days, the cultured BMSCs were harvested to analyze expression of targeted genes and proteins, respectively. The proliferation rate of BMSCs was also detected by cell counting kit-8 (CCK-8). In the in vivo experiments, the BMSCs-loaded thermogels with or without EMF pretreatment were first injected on HAC scaffolds at ambient temperature and incubated at $37^{\circ} \mathrm{C}$ for $30 \mathrm{~min}$. The prepared composite scaffolds were then implanted in osteochondral defect regions. After 12 weeks post implantation, histological and immunohistochemical analyses were performed to evaluate regenerated tissue. Meanwhile, subchondral bone was also assessed by micro-CT. These in vitro and in vivo results suggest that EMF has great potential to enhance osteochondral repair, especially cartilage regeneration; this provides the basis for further research and clinical translation of EMF for the repair of cartilage defects. 


\section{Materials And Methods}

\subsection{Preparation and characterization of PLGA-PEG-PLGA thermogel}

As reported in a previous study [43], the PLGA-PEG-PLGA copolymer was synthesized by ring-opening polymerization approach. Poly(ethylene glycol) (PEG) was dried under vacuum in a three-necked flask as the macroinitiator. Lactide $(113.46 \mathrm{~g})$ and glycolide $(30.48 \mathrm{~g})$ were added to the flask and mixed. After incubation of the mixture at $80^{\circ} \mathrm{C}$ for $30 \mathrm{~min}$, the reaction was activated by the catalyst stannous octoate $\left(\mathrm{Sn}(\mathrm{Oct})_{2}\right)$ at $\sim 150^{\circ} \mathrm{C}$. Subsequently, the unreacted monomers were removed by cooling down to $120^{\circ} \mathrm{C}$ under vacuum, and the crude copolymers were then washed in $80^{\circ} \mathrm{C}$ water three times to further eliminate water-soluble oligomers. After purification and drying, the copolymers were harvested and stored at $20^{\circ} \mathrm{C}$. PEG, LA, GA, and Sn(Oct) ${ }_{2}$ were purchased from Sigma-Aldrich (St. Louis, MO, USA). We also measured the sol-gel transition temperature via tube inverting method. The aqueous solution of copolymer $(0.5 \mathrm{~mL}, 25 \mathrm{wt} \%)$ was placed into a $2 \mathrm{~mL}$ centrifuge tube at $4^{\circ} \mathrm{C}$. Then, the tube was gradually heated in a water bath with a temperature increment of $1^{\circ} \mathrm{C}$ per step. After equilibration at each temperature, the tube was inverted to observe the liquidity of copolymer solution. When there was no evidence of flow for at least $30 \mathrm{~s}$, the temperature was recorded and determined as the sol-gel transition temperature.

\subsection{Preparation and characterization of HAC scaffold}

The porous scaffolds were manufactured from hydroxyapatite (HA) and monomeric Collagen type I (Col1) in accordance with our previous study [44]. The collagen was mixed with HA that had been dissolved in $\mathrm{HCl}$ to obtain a uniform $\mathrm{HAC}$ solution. By adjusting the $\mathrm{pH}$ value and temperature, the HAC solution would be collected after centrifugation and freeze drying. The produced scaffolds were cylindrical with a diameter of $4 \mathrm{~mm}$ and height of $5 \mathrm{~mm}$. In addition, we observed the microstructure of scaffolds via scanning electric microscopy (SEM; TESCAN VEGA 3, Brno, Czech Republic) and then analyzed their porosity, pore size, and compression strength.

\subsection{EMF device and exposure}

The EMF device has been described in our previous studies [41]. The equipment was designed and manufactured by the Naval Engineering University of China. The signals produced by a waveform generator were amplified through an amplifier and transmitted to the coils. An oscilloscope was used to observe and verify the frequency and intensity of signals. The layout diagram of EMF facility is shown in Fig. $1 \mathrm{H}$. On the basis of previous findings, we used a continuous EMF stimulation with parameters of 1 $\mathrm{mT}, 15 \mathrm{~Hz}$, and $4 \mathrm{~h} /$ day in this study [45]. The culture plates were placed in the center of Helmholtz coils, which were located in a humidified incubator with $5 \% \mathrm{CO}_{2}$ at $37^{\circ} \mathrm{C}$. The sham control samples were kept in another incubator under the same conditions without EMF exposure. 


\subsection{Isolation and culture of rabbit BMSCs}

BMSCs were isolated from iliac crest of New Zealand White rabbits. All procedures on animals were performed with approval from the Ethics and Animal Research Committee of Huazhong University of Science and Technology. The bone marrow and MSCs were harvested as described in the literature [46]. After anesthesia, bone marrow was aspirated via a small incision using an $18 \mathrm{G}$ needle and stored in a pre-heparinized syringe. Dulbecco's modified Eagle's medium (DMEM, Gibco), supplemented with 10\% fetal bovine serum (FBS) and 1\% penicillin and streptomycin, was added to marrow suspension. The isolated cells were cultured to adhere to the flask in a humidified incubator with $5 \% \mathrm{CO}_{2}$ at $37^{\circ} \mathrm{C}$. The culture media was first changed after five days and refreshed every three days thereafter. Adherent cells were detached and passaged when they reached $80 \%$ confluence. Third passage BMSCs were used in the subsequent experiments.

\subsection{Cell culture in PLGA-PEG-PLGA thermogel and cell viability assay}

After detachment and centrifugation, the rabbit BMSCs were resuspended in the PLGA-PEG-PLGA copolymer solution with a final concentration of $1.0 \times 10^{6} / \mathrm{mL}$. Subsequently, encapsulated BMSCs were placed in an incubator at $37^{\circ} \mathrm{C}$ for $30 \mathrm{~min}$ to achieve sol-to-gel transition. The expansion medium was added on the surface of gel and replaced by fresh medium every three days. The cell-encapsulated gels were prepared for following experiments. Further, the viability of BMSCs were assessed using a cell counting kit-8 (CCK-8, Boster, Wuhan, China) according to the standard protocol. BMSCs were seeded in thermogels and cultured for $1,4,7,10$, and 14 days. Then, a total of $180 \mu$ l culture medium, supplemented with $20 \mu \mathrm{L} \mathrm{CCK}-8$ solution, was added to each well in a 96-well plate. After incubation for $1.5 \mathrm{~h}$ at $37^{\circ} \mathrm{C}$, the absorbance was read at $450 \mathrm{~nm}$ by a microplate reader (Bio-Rad, Richmond, CA, United States).

\subsection{Quantitative reverse transcription-polymerase chain reaction (qRT-PCR)}

Total RNA was extracted using the EZNA Total RNA kit (Omega Bio-Tek, USA) and first strand cDNA was synthesized from $2 \mu \mathrm{g}$ RNA with the Reverse Transcription kit (Toyobo Life Science, Japan). The SYBR Green Real-Time PCR Master Mix (Toyobo Life Science, Japan) was used to quantify the expression of target genes on CFX96 (Bio-Rad Laboratories, USA) system. The relative expression levels of genes were normalized to corresponding GAPDH and analyzed by $2^{-\triangle \Delta C t}$ method. The sequences of primers used in this study were listed in Table $\mathbf{S 1}$. All the experiments were performed in triplicates.

\subsection{Western blot}

The total protein was extracted by radio immunoprecipitation assay lysis buffer (RIPA) supplemented with $1 \%$ protease and phosphatase inhibitors (Boster, Wuhan, China). The protein concentration was determined with a Bicinchoninic Acid Assay (BCA) protein assay kit (Boster, Wuhan, China). Then, $20 \mu \mathrm{g}$ 
protein samples were separated on 10\% SDS-PAGE and transferred onto PVDF membranes (Millipore, USA). First, the membranes were blocked for $1 \mathrm{~h}$ with $5 \%$ bovine serum albumin (BSA) at $37^{\circ} \mathrm{C}$. Second, membranes were incubated with appropriately diluted primary antibodies at $4^{\circ} \mathrm{C}$ overnight and subsequently washed for three times with Tris-buffered saline with $0.1 \%$ Tween-20 (TBST). Then, membranes were incubated with horseradish peroxidase (HRP)-conjugated secondary antibodies at room temperature for $1 \mathrm{~h}$. Finally, the protein bands were visualized under the Western enhanced chemiluminescence (ECL) system (Thermo Fisher Scientific, USA). The corresponding GAPDH bands were served as loading control and the amount of target proteins were semi-quantified by Image Lab system version 5.1 (Bio-Rad Laboratories, USA). All western blots were independently repeated three times, and the representative bands were obtained.

\subsection{Immunofluorescence}

The culture medium was removed and cells was washed three times with PBS. Then, cells were fixed with $4 \%$ paraformaldehyde for 15 min, permeabilized with $0.5 \%$ Triton X-100 for $15 \mathrm{~min}$, blocked with goat serum (the same species as secondary antibody) for $30 \mathrm{~min}$ at room temperature. In between these steps, cells were rinsed three times with PBS. Cells were incubated with primary antibodies with appropriate dilution at $4^{\circ} \mathrm{C}$ overnight, followed by fluorescein labeled secondary antibodies at room temperature for 1 $\mathrm{h}$ in dark. Additionally, nuclei were counterstained by DAPI. Fluorescent microscopy (EVOS FL Auto Imaging System, Life technologies, Gaithersburg, MD) was used to observe fluorescence and acquire images.

\subsection{Flow cytometry analysis}

BMSCs were prepared into single cell suspension, incubated with primary antibodies for $45 \mathrm{~min}$, and stained with secondary antibodies for $30 \mathrm{~min}$ at $4^{\circ} \mathrm{C}$. The corresponding isotype antibodies were used as negative control. The detection was performed by a flow cytometer (BD Biosciences, San Jose, CA, USA) and data analyzed by FlowJo software (FlowJo, LLC., Ashland, OR, USA)

\subsection{Rabbit model with femoral condyle defect and scaffold implantation}

A total of $80 \mathrm{New}$ Zealand rabbits (male, six-month-old) were used in this study. After anesthetization by intraperitoneal injection with $3 \%$ pentobarbital, the lateral femoral condyles were exposed and a $4 \mathrm{~mm}$ wide and $5 \mathrm{~mm}$ deep defect hole was created using a trephine $[47,48]$. The different sterile constructs that had been prepared were then implanted into the punch defects. The incisions were carefully sutured layer by layer. The rabbits were sacrificed at 4- and 12-weeks post operation for the following experiments. The animals were randomly divided into five groups, and each group consisted of eight rabbits: (1) "Blank" group, without construct implantation; (2) "HAC" group, HAC scaffold without thermogel; (3) "HAC + gel" group, HAC scaffold and acellular thermogel; (4) "HAC + gel + M" group, HAC scaffold and BMSCs-loaded thermogel; (5) "HAC + gel + M + E" group, HAC scaffold and BMSCs-loaded thermogel, where the BMSCs had been stimulated by EMF. 


\subsection{Biomechanical properties of repaired osteochondral tissue}

An Instron 5566 electromechanical testing device (Instron Corporation, USA) was applied to evaluate the biomechanical properties of regenerative osteochondral tissue at 12 weeks postoperatively. Repaired osteochondral samples with a thickness of $10 \mathrm{~mm}$ were cut from femoral condyle and then fixed on the plate. We used a custom stainless-steel rod (diameter $=4 \mathrm{~mm}$ ) to compress our samples with a strain rate of $0.01 \mathrm{~mm} / \mathrm{s}$. The maximum displacement was set at $0.5 \mathrm{~mm}$. The data recorded by a computer in real time were plotted as strain vs stress to capture the response of repaired osteochondral tissue under incremental load. The equilibrium Young's modulus $[49,50]$ was also calculated through such unconfined compression test at $5 \%$ axial strain.

\subsection{Microcomputed tomography (micro-CT) analysis}

The rabbits were euthanized to harvest specimens at 4 and 12 weeks after operation. The samples were then fixed for 2 days in $4 \%$ paraformaldehyde and scanned with micro-CT (Scanco, Brüttisellen, Switzerland) following standard protocol (70 kV, $114 \mu \mathrm{A}$, and $20 \mu \mathrm{m})$ [51]. Initial binary images were reconstructed to obtain 3D images using the built-in software. The bone mineral density (BMD) and the ratio of bone volume to total volume (BV/TV) within target regions were quantitatively evaluated.

\subsection{Histological evaluation}

The rabbits were sacrificed at 4- and 12-weeks post operation, and femurs were harvested. Specimens were fixed in $4 \%$ paraformaldehyde solution for two days. After fixation, samples were decalcified and embedded in paraffin blocks. Then, $5 \mu \mathrm{m}$ thick slices were produced for subsequent staining (including hematoxylin and eosin (H\&E), toluidine blue (T-B), safranin-fast green (S-F), and Masson's trichrome (MT)) to observe new-formed tissues. The histological scores were blindly and independently evaluated by three authors (Gaohong Sheng, JiYuan Yan and Hao Li) based on a well-established histological scoring system (Table S2 and S3) for osteochondral defects [52].

We also performed immunohistochemical (IHC) staining for chondrogenesis- and osteogenesis- related proteins, including Col2, aggrecan, Col X, Col1, and osteocalcin (OCN). The sections were immersed with $3 \%(\mathrm{w} / \mathrm{v}) \mathrm{H}_{2} \mathrm{O}_{2}$ and blocked with 5\% (w/v) BSA. After enzymatic antigen retrieval, sections were stained by primary antibodies at $4^{\circ} \mathrm{C}$ overnight, followed by incubation of horseradish peroxidase (HRP)conjugated secondary antibodies at room temperature for $50 \mathrm{~min}$. The newly prepared 3, 3diaminobenzidiine tetrahydrochloride (DAB) solution was added for visualization. Nuclei were counterstained with hematoxylin for 3 min.

\subsection{Statistical analysis}


All quantitative data were presented as mean \pm standard deviation (SD), unless otherwise specified, and ordinal data were shown as median (inter quartile range). For comparison between two groups, the Student's t-test was applied, while the statistical differences among groups were assessed through oneway analysis of variance (ANOVA) followed by the Tukey honestly significant difference (HSD) test. If equal variances are not assumed, Dunnett's test was used. In terms of ordinal data like histological scores, the Kruskal-Wallis test was used to analyze differences. A value of $p<0.05$ was considered statistically significant. SPSS 20.0 software (IMB Corp., USA) was used to perform statistical analyses.

\section{Results}

\subsection{Characterization of HAC scaffold and PLGA-PEG-PLGA thermogel}

As indicated in Fig. 1A, PLGA-PEG-PLGA copolymer appeared to be aqueous solution at $4^{\circ} \mathrm{C}$ and converted into gel state without fluidity when heated up to $37^{\circ} \mathrm{C}$ (Fig. 1B). Moreover, the sol-gel transition temperature of the copolymer was measured as $36^{\circ} \mathrm{C}$. The fabricated HAC scaffold presented a cylindrical shape with a diameter of $4 \mathrm{~mm}$ and height of $5 \mathrm{~mm}$ (Fig. 1C). The microstructure and morphology were further observed via SEM, and the images showed evenly distributed pores (Fig. 1D). The mean porosity was calculated as $77 \pm 5.79 \%$ (Fig. 1E), and the compression modulus was $13.46 \pm$ $0.88 \mathrm{MPa}$ (Fig. 1F). Given such a porous feature of HAC scaffold, the PLGA-PEG-PLGA aqueous solution could penetrate the scaffold at a relatively low temperature and be firmly adhered to HAC after completing sol-gel transition at $36^{\circ} \mathrm{C}$ (Fig. 1G).

\subsection{Evaluation of cell culture within thermogel}

The PLGA-PEG-PLGA hydrogel provided a three-dimensional environment for cell growth, which is distinct from the monolayer cell culture. Further, the interaction between cell and hydrogel matrix was weakened owing to the hydrophobicity of PEG chain segment. As shown in Fig. 2A, BMSCs within hydrogel presented a spherical morphology, while cells growing on plate with flat surface exhibited a typical spindle shape. The BMSCs were cultured in PLGA-PEG-PLGA hydrogel for live/dead staining at 1, 4, and 7 days. The results showed that hydrogel did not affect activity and proliferation of BMSCs. We also found the cells were fairly evenly distributed in hydrogel.

\subsection{EMF promotes proliferation of BMSCs partly by activating PI3K/AKT/mTOR pathway}

Live/dead staining revealed that the cell number in EMF group appeared to increase compared with control group, especially at Day 7 (Fig. 2A). To further assay the effect of EMF on cell proliferation, CCK-8 was performed at multiple points for a two-week period (Fig. 2B). The results showed that BMSCs proliferated over time in each group. Furthermore, there was no significant difference among groups in 
cell number within 1 week of EMF induction. However, EMF could significantly promote proliferation of BMSCs after stimulation for over ten days both in expansion medium (EM) and chondrogenic medium (CM) groups.

As indicated in Fig. 3A, EMF significantly enhanced the expression of proliferation-related genes, including Cyclin D1,CDK4, and PCNA. The protein levels of these genes in EMF groups were also increased when compared to the control group (Fig. 3A). We explored the underlying mechanisms by which EMF promoted cell proliferation. When cells cultured in expansion medium, EMF group showed a higher level in the gene expression of $A K T$ while no significant difference in PI3K and mTOR was observed, compared with the observations of the control group. However, in the case of using $\mathrm{CM}$, the gene expressions of PI3K, AKT, and mTOR were increased remarkably in the presence of EMF exposure (Fig. 3B). However, there was no notable difference in the total protein expressions of PI3K, AKT, and mTOR between EMF groups and corresponding control groups (Fig. 3B). The phosphorylation of these three proteins was enhanced by EMF stimulation and the phosphorylated proteins, detected by western blot analysis, were significantly increased both in the culture with EM and CM (Fig. 3B).

To verify whether EMF affects cell proliferation through PI3K/AKT/mTOR pathway, we used Dactolisib (PI3K inhibitor) and AZD8055 (mTOR inhibitor) to observe the changes of proliferation-related gene and protein expressions, respectively. The results of PCR analysis revealed significantly lower gene expressions of Cyclin D1, CDK4, and PCNA by PI3K inhibition in comparison with EMF group (Fig. 3C). The expression levels of these proteins were partly suppressed by addition of Dactolisib, compared with EMF stimulation (Fig. 3C). The similar results were also demonstrated when mTOR inhibition by AZD8055 (Fig. 3D).

\subsection{EMF enhanced chondrogenesis of BMSCs partly by activating Wnt1/LRP6/ $\beta$-catenin pathway}

First, we analyzed the distribution and expression of chondrogenesis-related proteins (including Col2, Aggrecan, and SOX9) by immunofluorescence after EMF exposure. Regarding to the distribution, the images indicted that SOX9 was expressed almost entirely within nucleus, while Col2 and Aggrecan were distributed both in the nucleus and cytoplasm. Notably, the expressions of these proteins were all significantly strengthened by EMF according to the analysis of fluorescence intensity (Fig. 4). Besides, we also detected the gene expressions of COL2, ACAN, and SOX9 by PCR. The EMF groups showed remarkably higher levels of these genes with a significant difference in comparison to control groups. The results of the western blot also revealed a remarkable increase in protein expressions (Fig. 5A).

Moreover, we investigated the potential mechanisms through which EMF played its prochondrogenic role in BMSCs. Both in EM and CM, the gene expression of WNT1, LRP6, and $\beta$-catenin in EMF groups were significantly higher than control groups. The analysis of protein expressions of Wnt1, LRP6, and $\beta$-catenin by western blot revealed similar trends (Fig. 5B). Then, the inhibitors of Wnt1 (SM04960) and $\beta$-catenin (LF3) were applied to demonstrate that EMF could promote chondrogenic differentiation of BMSCs by activating the Wnt1/LRP6/ $\beta$-catenin signaling pathway. The inhibition of Wnt1 could partially 
downregulate the increased gene expressions, which were induced by EMF. The protein expressions of Col2, Aggrecan, and SOX9 were also reduced dramatically by SM04960 (Fig. 5C). When using $\beta$-catenin inhibitor (LF3), the lower gene expressions of COL2, ACAN, and SOX9 were observed with statistically significance compared with EMF groups. Further, the protein expressions of Col2, Aggrecan, and SOX9 suggested a significant downregulation when inhibiting $\beta$-catenin with compared to EMF groups (Fig. $5 \mathrm{D})$.

\subsection{Evaluation for surface markers of rabbit BMSCs by flow cytometry}

As reported by Tan et al. [53], rabbit BMSCs positively expressed several surface markers, including CD81 and CD90. We used these two markers to further identify the isolated BMSCs and the results of flow cytometry showed that $96.9 \%$ and $97.5 \%$ of cells positively expressed CD81 (Fig. 6A) and CD90 (Fig. 6B), respectively. Further, the average fluorescence intensities of CD81 (Fig. 6C) and CD90 (Fig. 6D) were both gradually reduced with time in the culture with $\mathrm{CM}$. A remarkable decrease in CD81 fluorescence intensity was observed when BMSCs were stimulated by EMF for four days, and the decrease in CD 90 for five days. Although during chondrogenesis induction over a period of nine days, the number of cells positively expressing CD81 or CD90 was approximately the same. The peak fluorescence intensities were both decreased with time. Furthermore, the lower intensity in EMF group indicated that EMF could accelerate the peak fluorescence intensity of CD81 and CD90 to shift to the left.

\subsection{Evaluation of regenerated tissue by macroscopic observation}

A rabbit model (Fig. 7A) with a critical-sized defect was used to assess the effect of EMF on osteochondral repair in vivo. As shown in (Fig. 7B), there was no obvious repair in the Blank group, and only a small amount of chondroid new-formed tissue was found around scaffold in HAC and HAC + gel groups. With regard to HAC + gel + MSCs group, the defect was filled with more cartilage-like tissue, although it was less transparent than that in EMF group. From 4 to 12 weeks after implantation, the defect repair was significantly enhanced in last four groups, while degradation was observed at the defect site in Blank group. Around the edge of defects, regenerated tissues were found both in HAC and $\mathrm{HAC}+$ gel groups. Besides, the HAC + gel group indicated a thin layer of cartilaginous tissue covering the defect hole. When BMSCs were planted within the thermogel, the new-formed tissue was remarkably increased. However, a clear boundary between the native and new tissue suggested an incomplete repair with poor lateral integration. Notably, this boundary was almost eliminated by application of EMF, and the repaired tissue showed a similar appearance to native hyaline cartilage.

\subsection{Evaluation of mechanical properties of regenerated tissue by unconfined compression}

Page $11 / 34$ 
We recorded the data of axial deformation and corresponding pressure and plotted the strain-stress curve based on representative points (Fig. 7C). Evidently, the EMF group showed a lowest strain under the same stress, which indicated the new-formed tissue in the EMF group possessed a satisfactory mechanical property with highest strength. Young's modulus was also calculated at $5 \%$ axial strain in each group (Fig. 7D). The normal osteochondral tissue had a modulus above $2 \mathrm{MPa}$. The modulus of repaired tissue in EMF group was significantly higher than in the other three groups. These results suggested that EMF could significantly improve the biomechanical properties of new-formed osteochondral tissue.

\subsection{Evaluation of regenerated tissue by Micro-CT analysis}

Micro-CT was further performed to characterize regenerated tissue by reconstruction of 3D images and analysis of statistical data. For the Blank group, there was little self-repair bone with a large cavity. In terms of defect surface, images showed that regenerative bone in EMF group was the densest with good integration to adjacent native tissue. The amount of new bone repaired by the cellular scaffold was also significantly increased compared with the cell-free groups. The largest amount of new bone was observed in the EMF group (Fig. 8A). The bone volume per total volume (BV/TV) was highest in the EMF group in both the trabecular volume of interest (VOI) and cartilage and cortical (C\&C) VOI when compared to other groups. The same trend was also shown for BMD for both trabecular VOI and C\&C VOI (Fig. 8B).

\subsection{Histological evaluation of regenerated tissue}

Histological staining was performed to confirm the effect of EMF on promoting osteochondral repair, especially for the repair of damaged cartilage (Fig. 9). In cell-loaded groups, a layer of new-formed cartilage was observed within the chondral defect region. Furthermore, staining of both toluidine blue (TB) and safranin O-fast green (S-F) indicates that the new cartilage in $\mathrm{HAC}+$ gel $+\mathrm{M}+\mathrm{E}$ group is significantly flatter and thicker with a better integration to adjacent native cartilage, compared to the HAC + gel $+\mathrm{M}$ group. We also found a pattern of chondrocytes arrangement within new cartilage similar to that of native cartilage in EMF group, which appeared to be more orderly and aligned.

The histological staining was further assessed by using a quantitative scoring system. In terms of overall defect evaluation, the same scores for overall filling and overall degradation suggested there was a dynamic balance between tissue regeneration and scaffold degradation (Fig. 10A). Significant differences for both overall filling and degradation were found only in EMF group, but not in other three groups when compared to the Blank group. For subchondral bone evaluation, the scores of bone filling, bone morphology, and bone bonding were all highest in the EMF group (Fig. 10B). Although the bone morphology score in $\mathrm{HAC}+$ gel $+\mathrm{M}$ was also dramatically higher than that in Blank group. In terms of cartilage evaluation, all parameters remained the highest in EMF group and exhibited significant differences when compared with Blank group (Fig. 10C). 


\subsection{Immunohistochemical staining of regenerated tissue}

Several cartilage-specific proteins were stained by immunohistochemical methods to further evaluate the new cartilage (Fig. 11A). The positive staining regions were matched with those in T-B and S-F staining. In the last two groups with BMSCs, the staining of Col2 and Aggrecan were both positive, while there was almost no positive staining in cell-free groups. The stronger staining of these two proteins suggested an increase in the protein expression of the EMF group above the one in the HAC + gel + M group. The most positive chondrocytes within the chondral defect were observed in the EMF group, with respect to both Col2 and Aggrecan. The expression of Col $\mathrm{X}$ indicates the hypertrophy and calcification of chondrocytes. Col $\mathrm{X}$ was relatively reduced in EMF group compared to HAC + gel $+\mathrm{M}$ group. $\mathrm{OCN}$ and Col1 were also stained for evaluation of subchondral bone repair (Fig. 11B). The positive staining was negligible in the first two groups, while both proteins were evident in last three groups. Moreover, the strongest staining and most positive cells in EMF group demonstrated that EMF could also enhance subchondral bone repair.

\section{Discussion}

Articular cartilage repair remains a significant challenge for researchers and clinicians, especially in the reconstruction of hyaline cartilage with proper biomechanical function [54,55]. Compared to other methods of cartilage repair, such as bone marrow stimulation and autografts implantation, cartilage tissue engineering appears to be the most attractive therapy. With the development of new biomaterials and fabrication techniques, a variety of scaffolds have emerged with hierarchical structures, excellent mechanical properties, and biological performance[56, 57]. Such scaffolds provide a three-dimensional microenvironment for growth and migration of cells derived from diverse sources that have the potential to differentiate into chondrocytes [5]. Numerous scaffolds have been investigated for cartilage reconstruction, among which the polymeric materials attracted significant attention, primarily in forms of sponges, fibrous meshes, and hydrogels. The injectable thermosensitive hydrogels can easily fill the cartilage defect with irregular shape and be transplanted by a minimally invasive procedure. More importantly, the cells could be suspended homogenously within thermogels in a sol state and proliferate without loss of differentiation potential [58]. Hydrogels simulate physiological ECM surrounding chondrocytes and transmit external stress to cells encapsulated in gels. After sol-to-gel transition, hydrogels also obtain certain mechanical strength, although such mechanics are limited. In addition, the defect of cartilage is often accompanied by the damage of subchondral bone, hence the repair of subchondral bone is key to cartilage regeneration. We employed the HAC scaffold to repair the bone defects in the previous study. Thereby, a composite scaffold consisting of HAC and PLGA-PEG-PLGA thermogel was produced to repair the osteochondral defect in this study. The repair requirements for articular cartilage and subchondral bone are different owing to their distinct physiological features and functions. Our results show that it was feasible to reconstruct cartilage and subchondral bone by two types of materials, which were suitable for repairing cartilage and bone, respectively. 
Biophysical stimulations exert significant effects on cellular behavior, and some have been applied in clinical practice [59]. In addition to chemical factors, physical signals also play a regulatory role in the development and regeneration of bone and cartilage. The Food and Drug Administration (FDA) has approved the use of pulsed electromagnetic fields (PEMFs) for the treatment of delayed union and nonunion of bone fractures. Although increasing evidence suggested that PEMF could be an alternative therapy for cartilage repair, clinical application of PEMF in joints is still debated and more research is needed [60]. A narrative review summarized the current research involving the effect of EMF on articular cartilage and potential application in joint diseases [38]. The stimulation of PEMF could increase intracellular calcium concentration by regulating calcium channels, which are required for the chondrogenic differentiation of MSCs [31]. When the parameters of EMF were tuned to the cyclotron resonance frequencies of calcium ions, Kavand et al. reported an enhanced efficacy of chondrogenesis [33]. Recently, one study showed that the MSC-derived conditioned medium post EMF exposure was also capable to promote cartilage regeneration and demonstrated that EMF could regulate the paracrine function of MSCs [36]. Previous studies have shown that Wnt1/ $\beta$-catenin signaling pathway is regulated by EMF in osteogenesis and bone metabolism $[61,62]$. Based on our results in this study, we further verified that EMF could also facilitate chondrogenic differentiation and contribute to improving cartilage repair by activating the Wnt1/LRP6/ $\beta$-catenin pathway.

The cell proliferation as well as ECM synthesis are both crucial in the cartilage regeneration. The positive effect of EMF on cell proliferation has been observed in various cell types, such as MSCs [63], chondrocytes [64], and osteoblasts.[65] Consistently with previous results, our study further demonstrated that EMF could promote proliferation of BMSCs by upregulating PI3K/Akt/mTOR pathway. Nevertheless, some studies indicate that EMF has no effects on cell proliferation[66, 67].The cell types and EMF parameters may together account for these inconsistent results. More importantly, the cartilage defects would cause joint inflammation, which in turn exacerbates cartilage deterioration. Breaking this vicious cycle will significantly contribute to cartilage repair. Numerous studies have elaborated on the possible mechanisms that EMF alleviates inflammatory response. For instance, the anti-inflammatory effect of EMF appeared to be mediated by increasing adenosine receptors like $A_{2} A$ and $A_{3}$ [68-70]. In mice with osteoarthritis (OA), EMF inhibited the expression of inflammatory cytokines, including IL-1 $\beta$, ADAMTS4, and MMP13 [71]. Moreover, the effects of EMF on inflammation and underlying mechanisms have been systematically reviewed in terms of OA [72] or RA [39]. Although the application of EMF to treat OA or RA must be verified by more clinical trials with long-term follow-up, it is also one of the possible mechanisms by which EMF enhances cartilage repair.

For tissue repair, there have been many preclinical and clinical studies on the role of EMF in bone repair, while only few addressed cartilage regeneration [37, 73]. A review depicted the potential benefits of EMF in cartilage repair [70]. To develop therapeutic strategy combined with EMF, it is necessary to optimize EMF parameters, such as the waveform, field direction, intensity, frequency, and time of exposure. However, the combinations of above parameters are theoretically innumerable, and there is no experiment design that can cover all these parameters. Thus, the community of EMF research must focus on 
unveiling the underlying laws and mechanisms, by which EMF exerts its biological effects. Moreover, the type of recipient cells or tissues stimulated by EMF is another essential factor that affects the quality of tissue repair [74]. With the advances of novel physical stimulus modalities, the application of EMF in medicine will be further broadened, and this effect will be reinforced. Several studies have introduced magnetic beads that can feel the force of EMF to biological culture system. Song et al. reported that the magnetic beads could promote cell proliferation driven by EMF. TGF- $\beta$-immobilized magnetic beads successfully induced MSCs to generate cartilage in vitro under magnetic forces $[75,76]$. Moreover, EMF can operate without direct contact and thus enable extracorporeal control.

The articular cartilage defect is a common medical problem that severely affects the life quality of patients. The therapeutical strategies currently used in clinical practice relief associated symptoms and yield acceptable outcomes in the short term. However, the repaired cartilage was found to degrade during a long period of follow-up, as new-formed tissue was often fibrocartilage rather than hyaline-like cartilage [77]. Thus, the ambitious goal of cartilage repair is to regenerate hyaline cartilage with proper mechanical function and prevent long-term degradation. To the best of our knowledge, our study was the first to demonstrate that sinusoidal EMF can significantly improve cartilage regeneration when combined with tissue engineering. We also explored the underlying mechanisms and found that PI3K/AKT/mTOR and Wnt1/LRP6/ $\beta$-catenin signaling pathways mediated the transduction of biological effects of EMF for cell proliferation and differentiation, respectively. Furthermore, the lateral integration of repair cartilage with adjacent native tissue was improved, which may further contribute to the effect of EMF on cartilage repair. Furthermore, EMF could also enhance bone repair, which has been extensively studied. In short, EMF plays an essential role in the repair of osteochondral defects, and more research is required to explore optimal parameters and underlying mechanisms, especially for cartilage regeneration. These encouraging results from our study can pave the way for the application of biophysical stimuli in clinical cartilage repair.

\section{Conclusion}

A composite scaffold was constructed using a HAC and PLGA-PEG-PLGA thermogel, which matched with cartilage and subchondral layers in osteochondral defects, respectively. The BMSCs encapsulated in the thermogel were stimulated by EMF. Results from in vitro experiments suggested that EMF could promote proliferation and chondrogenic differentiation of BMSCs by activating the PI3K/AKT/mTOR and Wnt1/LRP6/ $\beta$-catenin pathways, respectively. In vivo evaluation further confirmed that EMF could enhance repair of osteochondral defects, especially cartilage repair, combined with tissue engineering in rabbits. Therefore, this study significantly advances the potential application of EMF or even other biophysical stimuli towards tissue regeneration.

\section{Abbreviations}

$\mathrm{BMD}$, bone mineral density; $\mathrm{BMSC}$, bone marrow mesenchymal stem cells; $\mathrm{CM}$, chondrogenic medium; EM, expansion medium; EMF, electromagnetic field; H\&E, hematoxylin and eosin; PEMF, pulsed 
electromagnetic fields; VOI, volume of interest.

\section{Declarations}

\section{Acknowledgments}

Not applicable.

\section{Author contributions}

J. Yan, C. Liu, Y. Zhang, C. Tu, R. Zhang, X. Tang, H. Li, H. Wang, and Y. Ma performed the experiments, analyzed the data and wrote the manuscript, H. Wu, G. Sheng supervised the project and edited the manuscript. All authors reviewed the manuscript.

\section{Funding}

This work was financially supported by the National Natural Science Foundation of China (No. 51537004 , No. 51877097, No. 51907077).

\section{Availability of data and materials}

The datasets used and/or analyzed during the current study are available from the corresponding author on reasonable request.

\section{Ethics approval and consent to participate}

All animal experimental procedures were complying with the Guidelines of Animal Care and Use Committee for Teaching and Research of Huazhong University of Science and Technology. The experimental protocol was approved by the committee. All efforts were conducted to minimize animal suffering.

\section{Consent for publication}

Not applicable.

\section{Conflict of interest}

The authors have no financial disclosures or conflicts of interest with the research

presented.

\section{Author details}

${ }^{1}$ Department of Orthopedics, Tongji Hospital, Tongji Medical College, Huazhong University of Science and Technology, Wuhan, Hubei, PR China. ${ }^{2}$ Department of Traumatology, Tongji Hospital, Tongji Medical 
College, Huazhong University of Science and Technology, Wuhan, Hubei, PR China. ${ }^{3}$ Department of Orthopedics, Renmin Hospital of Wuhan University, Wuhan, Hubei, PR China. ${ }^{4}$ Department of Radiology, Tongji Hospital, Tongji Medical College, Huazhong University of Science and Technology, Wuhan, Hubei, PR China. ${ }^{5}$ Department of Spine and Spinal Cord Surgery, Henan Provincial People's Hospital, People's Hospital of Zhengzhou University, HenanखZhengzhou, PR China. ${ }^{6}$ Department of Orthopedics, Shanxi Bethune Hospital, Taiyuan, Shanxi, PR China

\section{References}

1. Muir $\mathrm{H}$. The chondrocyte, architect of cartilage. Biomechanics, structure, function and molecular biology of cartilage matrix macromolecules. Bioessays. 1995;17(12):1039-48.

2. Gratz KR, Wong BL, Bae WC, et al. The effects of focal articular defects on cartilage contact mechanics. J Orthop Res. 2009;27(5):584-92.

3. Heidari B. Knee osteoarthritis prevalence, risk factors, pathogenesis and features: Part I. Caspian J Intern Med. 2011;2(2):205-12.

4. Hunziker EB. Articular cartilage repair: basic science and clinical progress. A review of the current status and prospects. Osteoarthritis Cartilage. 2002;10(6):432-63.

5. Driessen BJH, Logie C, Vonk LA. Cellular reprogramming for clinical cartilage repair. Cell Biol Toxicol. 2017;33(4):329-49.

6. Huey DJ, Hu JC, Athanasiou KA. Unlike bone, cartilage regeneration remains elusive. Science. 2012;338(6109):917-21.

7. Chu CR, Fortier LA, Williams A, et al. Minimally Manipulated Bone Marrow Concentrate Compared with Microfracture Treatment of Full-Thickness Chondral Defects: A One-Year Study in an Equine Model. J Bone Joint Surg Am. 2018;100(2):138-46.

8. Gobbi A, Karnatzikos G, Kumar A. Long-term results after microfracture treatment for full-thickness knee chondral lesions in athletes. Knee Surg Sports Traumatol Arthrosc. 2014;22(9):1986-96.

9. Brittberg M, Lindahl A, Nilsson A, et al. Treatment of deep cartilage defects in the knee with autologous chondrocyte transplantation. N Engl J Med. 1994;331(14):889-95.

10. Filardo G, Vannini F, Marcacci M, et al. Matrix-assisted autologous chondrocyte transplantation for cartilage regeneration in osteoarthritic knees: results and failures at midterm follow-up. Am J Sports Med. 2013;41(1):95-100.

11. Salzmann GM, Sauerschnig M, Berninger MT, et al. The dependence of autologous chondrocyte transplantation on varying cellular passage, yield and culture duration. Biomaterials. 2011;32(25):5810-8.

12. Marcacci M, Kon E, Delcogliano M, et al. Arthroscopic autologous osteochondral grafting for cartilage defects of the knee: prospective study results at a minimum 7-year follow-up. Am J Sports Med. 2007;35(12):2014-21. 
13. Smith GD, Knutsen G, Richardson JB. A clinical review of cartilage repair techniques. J Bone Joint Surg Br. 2005;87(4):445-9.

14. Temenoff JS, Mikos AG. Review: tissue engineering for regeneration of articular cartilage. Biomaterials. 2000;21(5):431-40.

15. Yang HS, La WG, Bhang SH, et al. Hyaline cartilage regeneration by combined therapy of microfracture and long-term bone morphogenetic protein-2 delivery. Tissue Eng Part A. 2011;17(1314):1809-18.

16. Mithoefer K, McAdams T, Williams RJ, et al. Clinical efficacy of the microfracture technique for articular cartilage repair in the knee: an evidence-based systematic analysis. Am J Sports Med. 2009;37(10):2053-63.

17. Hangody L, Dobos J, Balo E, et al. Clinical experiences with autologous osteochondral mosaicplasty in an athletic population: a 17-year prospective multicenter study. Am J Sports Med. 2010;38(6):1125-33.

18. Moseley JB Jr, Anderson AF, Browne JE, et al. Long-term durability of autologous chondrocyte implantation: a multicenter, observational study in US patients. Am J Sports Med. 2010;38(2):23846.

19. Emre TY, Ege T, Kose O, et al. Factors affecting the outcome of osteochondral autografting (mosaicplasty) in articular cartilage defects of the knee joint: retrospective analysis of 152 cases. Arch Orthop Trauma Surg. 2013;133(4):531-6.

20. Mahmoudifar N, Doran PM. Tissue engineering of human cartilage and osteochondral composites using recirculation bioreactors. Biomaterials. 2005;26(34):7012-24.

21. Makris EA, Gomoll AH, Malizos KN, et al. Repair and tissue engineering techniques for articular cartilage. Nat Rev Rheumatol. 2015;11(1):21-34.

22. Moon HJ, Ko du Y, Park MH, et al. Temperature-responsive compounds as in situ gelling biomedical materials. Chem Soc Rev. 2012;41(14):4860-83.

23. Yan Q, Xiao LQ, Tan L, et al. Controlled release of simvastatin-loaded thermo-sensitive PLGA-PEGPLGA hydrogel for bone tissue regeneration: in vitro and in vivo characteristics. J Biomed Mater Res A. 2015;103(11):3580-9.

24. Cao D, Zhang X, Akabar MD, et al. Liposomal doxorubicin loaded PLGA-PEG-PLGA based thermogel for sustained local drug delivery for the treatment of breast cancer. Artif Cells Nanomed Biotechnol. 2019;47(1):181-91.

25. Gao Y, Sun Y, Ren F, et al. PLGA-PEG-PLGA hydrogel for ocular drug delivery of dexamethasone acetate. Drug Dev Ind Pharm. 2010;36(10):1131-8.

26. Santovena A, Monzon C, Alvarez-Lorenzo C, et al. Structure-Performance Relationships of Temperature-Responsive PLGA-PEG-PLGA Gels for Sustained Release of Bone Morphogenetic Protein-2. J Pharm Sci. 2017;106(11):3353-62.

27. Liu H, Ding J, Li C, et al. Hydrogel is Superior to Fibrin Gel as Matrix of Stem Cells in Alleviating Antigen-Induced Arthritis. Polymers (Basel) 2016;8(5). 
28. Zhang Y, Zhang J, Chang F, et al. Repair of full-thickness articular cartilage defect using stem cellencapsulated thermogel. Mater Sci Eng C Mater Biol Appl. 2018;88:79-87.

29. Oldershaw RA. Cell sources for the regeneration of articular cartilage: the past, the horizon and the future. Int J Exp Pathol. 2012;93(6):389-400.

30. Liu X, Fu X, Dai G, et al. Comparative analysis of curative effect of bone marrow mesenchymal stem cell and bone marrow mononuclear cell transplantation for spastic cerebral palsy. J Transl Med. 2017;15(1):48.

31. Uzieliene I, Bernotas P, Mobasheri A, et al. The Role of Physical Stimuli on Calcium Channels in Chondrogenic Differentiation of Mesenchymal Stem Cells. Int J Mol Sci 2018;19(10).

32. Parate D, Franco-Obregon A, Frohlich J, et al. Enhancement of mesenchymal stem cell chondrogenesis with short-term low intensity pulsed electromagnetic fields. Sci Rep. 2017;7(1):9421.

33. Kavand $H$, van Lintel $H$, Renaud $P$. Efficacy of pulsed electromagnetic fields and electromagnetic fields tuned to the ion cyclotron resonance frequency of $\mathrm{Ca}(2+)$ on chondrogenic differentiation. $\mathrm{J}$ Tissue Eng Regen Med. 2019;13(5):799-811.

34. Aaron RK, Wang S, Ciombor DM. Upregulation of basal TGFbeta1 levels by EMF coincident with chondrogenesis-implications for skeletal repair and tissue engineering. $J$ Orthop Res. 2002;20(2):233-40.

35. Jansen JH, van der Jagt OP, Punt BJ, et al. Stimulation of osteogenic differentiation in human osteoprogenitor cells by pulsed electromagnetic fields: an in vitro study. BMC Musculoskelet Disord. 2010;11:188.

36. Parate D, Kadir ND, Celik C, et al. Pulsed electromagnetic fields potentiate the paracrine function of mesenchymal stem cells for cartilage regeneration. Stem Cell Res Ther. 2020;11(1):46.

37. Stefani RM, Barbosa S, Tan AR, et al. Pulsed electromagnetic fields promote repair of focal articular cartilage defects with engineered osteochondral constructs. Biotechnol Bioeng. 2020;117(5):158496.

38. Vicenti G, Bizzoca D, Nappi VS, et al. Biophysical stimulation of the knee with PEMFs: from bench to bedside. J Biol Regul Homeost Agents. 2018;32(6 Suppl. 1):23-8.

39. Ross CL, Ang DC, Almeida-Porada G. Targeting Mesenchymal Stromal Cells/Pericytes (MSCs) With Pulsed Electromagnetic Field (PEMF) Has the Potential to Treat Rheumatoid Arthritis. Front Immunol. 2019;10:266.

40. Yin Y, Chen P, Yu Q, et al. The Effects of a Pulsed Electromagnetic Field on the Proliferation and Osteogenic Differentiation of Human Adipose-Derived Stem Cells. Med Sci Monit. 2018;24:3274-82.

41. Wang $H$, Tang $X$, Li W, et al. Enhanced osteogenesis of bone marrow stem cells cultured on hydroxyapatite/collagen I scaffold in the presence of low-frequency magnetic field. J Mater Sci Mater Med. 2019;30(8):89.

42. Tu $\mathrm{C}, \mathrm{Xiao} Y, \mathrm{Ma} Y$, et al. The legacy effects of electromagnetic fields on bone marrow mesenchymal stem cell self-renewal and multiple differentiation potential. Stem Cell Res Ther. 2018;9(1):215. 
43. Chen Y, Shi J, Zhang Y, et al. An injectable thermosensitive hydrogel loaded with an ancient natural drug colchicine for myocardial repair after infarction. J Mater Chem B. 2020;8(5):980-92.

44. Liu C, Abedian R, Meister R, et al. Influence of perfusion and compression on the proliferation and differentiation of bone mesenchymal stromal cells seeded on polyurethane scaffolds. Biomaterials. 2012;33(4):1052-64.

45. Yong Y, Ming ZD, Feng $L$, et al. Electromagnetic fields promote osteogenesis of rat mesenchymal stem cells through the PKA and ERK1/2 pathways. J Tissue Eng Regen Med. 2016;10(10):E537-45.

46. Zellner J, Pattappa G, Koch M, et al. Autologous mesenchymal stem cells or meniscal cells: what is the best cell source for regenerative meniscus treatment in an early osteoarthritis situation? Stem Cell Res Ther. 2017;8(1):225.

47. Link DP, van den Dolder J, van den Beucken JJ, et al. Bone response and mechanical strength of rabbit femoral defects filled with injectable CaP cements containing TGF-beta 1 loaded gelatin microparticles. Biomaterials. 2008;29(6):675-82.

48. Ahern BJ, Parvizi J, Boston R, et al. Preclinical animal models in single site cartilage defect testing: a systematic review. Osteoarthritis Cartilage. 2009;17(6):705-13.

49. Korhonen RK, Laasanen MS, Toyras J, et al. Comparison of the equilibrium response of articular cartilage in unconfined compression, confined compression and indentation. J Biomech. 2002;35(7):903-9.

50. Park S, Hung CT, Ateshian GA. Mechanical response of bovine articular cartilage under dynamic unconfined compression loading at physiological stress levels. Osteoarthritis Cartilage. 2004;12(1):65-73.

51. Qi X, Li H, Qiao B, et al. Development and characterization of an injectable cement of nano calciumdeficient hydroxyapatite/multi(amino acid) copolymer/calcium sulfate hemihydrate for bone repair. Int J Nanomedicine. 2013;8:4441-52.

52. Lu S, Lam J, Trachtenberg JE, et al. Dual growth factor delivery from bilayered, biodegradable hydrogel composites for spatially-guided osteochondral tissue repair. Biomaterials. 2014;35(31):8829-39.

53. Tan SL, Ahmad TS, Selvaratnam L, et al. Isolation, characterization and the multi-lineage differentiation potential of rabbit bone marrow-derived mesenchymal stem cells. J Anat. 2013;222(4):437-50.

54. Khan IM, Gilbert SJ, Singhrao SK, et al. Cartilage integration: evaluation of the reasons for failure of integration during cartilage repair. A review. Eur Cell Mater. 2008;16:26-39.

55. Bedi A, Feeley BT, Williams RJ. 3rd. Management of articular cartilage defects of the knee. J Bone Joint Surg Am. 2010;92(4):994-1009.

56. Du Y, Liu H, Yang Q, et al. Selective laser sintering scaffold with hierarchical architecture and gradient composition for osteochondral repair in rabbits. Biomaterials. 2017;137:37-48.

57. Galarraga JH, Kwon MY, Burdick JA. 3D bioprinting via an in situ crosslinking technique towards engineering cartilage tissue. Sci Rep. 2019;9(1):19987. 
58. An YH, Webb D, Gutowska A, et al. Regaining chondrocyte phenotype in thermosensitive gel culture. Anat Rec. 2001;263(4):336-41.

59. Massari L, Benazzo F, Falez F, et al. Biophysical stimulation of bone and cartilage: state of the art and future perspectives. Int Orthop. 2019;43(3):539-51.

60. Bjordal JM, Johnson MI, Lopes-Martins RA, et al. Short-term efficacy of physical interventions in osteoarthritic knee pain. A systematic review and meta-analysis of randomised placebo-controlled trials. BMC Musculoskelet Disord. 2007;8:51.

61. Catalano A, Loddo S, Bellone F, et al. Pulsed electromagnetic fields modulate bone metabolism via RANKL/OPG and Wnt/beta-catenin pathways in women with postmenopausal osteoporosis: A pilot study. Bone. 2018;116:42-6.

62. Zhang B, Xie Y, Ni Z, et al. Effects and Mechanisms of Exogenous Electromagnetic Field on Bone Cells: A Review. Bioelectromagnetics. 2020;41(4):263-78.

63. Fan W, Qian F, Ma Q, et al. $50 \mathrm{~Hz}$ electromagnetic field exposure promotes proliferation and cytokine production of bone marrow mesenchymal stem cells. Int J Clin Exp Med. 2015;8(5):7394-404.

64. Escobar JF, Vaca-Gonzalez JJ, Guevara JM, et al. In Vitro Evaluation of the Effect of Stimulation with Magnetic Fields on Chondrocytes. Bioelectromagnetics. 2020;41(1):41-51.

65. Suryani L, Too JH, Hassanbhai AM, et al. Effects of Electromagnetic Field on Proliferation, Differentiation, and Mineralization of MC3T3 Cells. Tissue Eng Part C Methods. 2019;25(2):114-25.

66. Ciombor DM, Lester G, Aaron RK, et al. Low frequency EMF regulates chondrocyte differentiation and expression of matrix proteins. J Orthop Res. 2002;20(1):40-50.

67. De Mattei M, Pellati A, Pasello M, et al. Effects of physical stimulation with electromagnetic field and insulin growth factor-l treatment on proteoglycan synthesis of bovine articular cartilage. Osteoarthritis Cartilage. 2004;12(10):793-800.

68. Varani K, De Mattei M, Vincenzi F, et al. Characterization of adenosine receptors in bovine chondrocytes and fibroblast-like synoviocytes exposed to low frequency low energy pulsed electromagnetic fields. Osteoarthritis Cartilage. 2008;16(3):292-304.

69. Vincenzi $F$, Targa $M$, Corciulo $C$, et al. Pulsed electromagnetic fields increased the anti-inflammatory effect of $A(2) A$ and $A(3)$ adenosine receptors in human T/C-28a2 chondrocytes and hFOB 1.19 osteoblasts. PLoS One. 2013;8(5):e65561.

70. Iwasa K, Reddi AH. Pulsed Electromagnetic Fields and Tissue Engineering of the Joints. Tissue Eng Part B Rev. 2018;24(2):144-54.

71. Ye W, Guo H, Yang X, et al. Pulsed Electromagnetic Field Versus Whole Body Vibration on Cartilage and Subchondral Trabecular Bone in Mice With Knee Osteoarthritis. Bioelectromagnetics. 2020;41(4):298-307.

72. Wang T, Xie W, Ye W, et al. Effects of electromagnetic fields on osteoarthritis. Biomed Pharmacother. 2019;118:109282. 
73. Chang $\mathrm{CH}$, Loo ST, Liu HL, et al. Can low frequency electromagnetic field help cartilage tissue engineering? J Biomed Mater Res A. 2010;92(3):843-51.

74. Fini M, Pagani S, Giavaresi G, et al. Functional tissue engineering in articular cartilage repair: is there a role for electromagnetic biophysical stimulation? Tissue Eng Part B Rev. 2013;19(4):353-67.

75. Motoyama M, Deie M, Kanaya A, et al. In vitro cartilage formation using TGF-beta-immobilized magnetic beads and mesenchymal stem cell-magnetic bead complexes under magnetic field conditions. J Biomed Mater Res A. 2010;92(1):196-204.

76. Song SH, Choi J, Jung HI. A microfluidic magnetic bead impact generator for physical stimulation of osteoblast cell. Electrophoresis. 2010;31(16):2762-70.

77. Mollon B, Kandel R, Chahal J, et al. The clinical status of cartilage tissue regeneration in humans. Osteoarthritis Cartilage. 2013;21(12):1824-33.

\section{Figures}
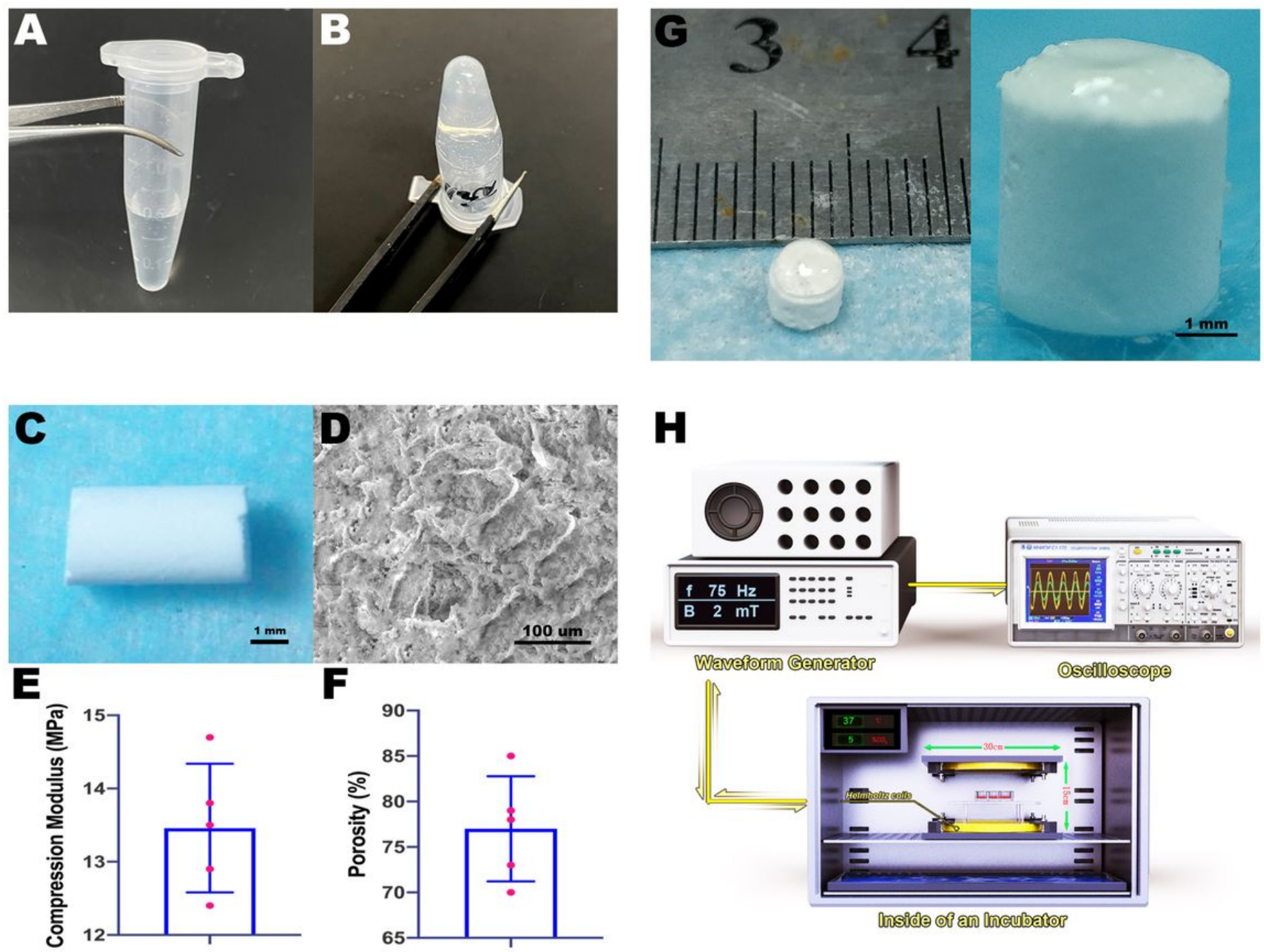


\section{Figure 1}

Characterization of composite scaffold and schematic diagram of EMF device. (A) Thermo-sensitive PLGA-PEG-PLGA copolymers exhibited a free-flowing sol at ambient temperature $\left(25^{\circ} \mathrm{C}\right)$ and $(\mathrm{B})$ spontaneously converted into a semi-solid hydrogel at body temperature $\left(36^{\circ} \mathrm{C}\right)$. (C) Macroscopic observation of cylindrical HAC scaffold produced by hydroxyapatite and collagen I. (D) Image of scanning electron micrograph (SEM) showing porous microstructure of HAC. (E) Compression modulus of HAC scaffold. (F) Porosity of HAC scaffold. (G) Assembly of composite scaffold. The cell-loaded aqueous sol was injected on the top of HAC and then incubated at $37^{\circ} \mathrm{C}$ for $30 \mathrm{~min}$. (H) Presentation of electromagnetic fields (EMF) device consisting of waveform generator, oscilloscope, and Helmholtz coils. Cells were stimulated at the center of coils, which was placed in an incubator at $37^{\circ} \mathrm{C}$ and $5 \% \mathrm{CO} 2$. 

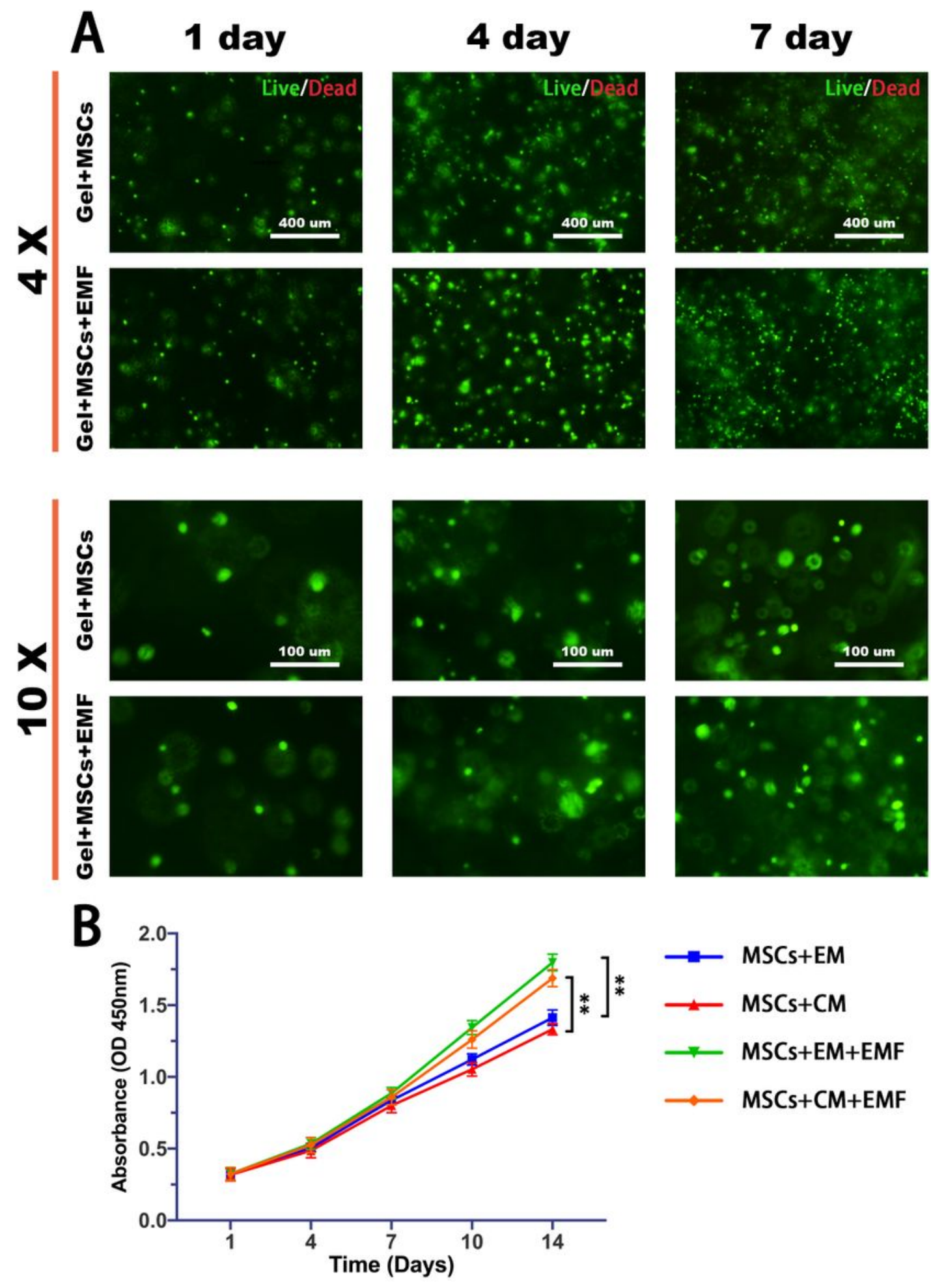

Figure 2

Cell culture and proliferation in hydrogel with or without EMF. (A) Images of Live/Dead staining demonstrate that the hydrogel supports cell viability and expansion of BMSCs after culturing for one, four, and seven days (green: live cells, red: dead cells). The cells were evenly distributed in the gel. (B) BMSCs proliferated over time, and EMF could significantly promote cell proliferation both in expansion 
medium (EM) and chondrogenic medium (CM). Data are shown as mean \pm standard deviation $(n=5)$. \# indicates no significant difference, ${ }^{*} p<0.05,{ }^{*} p<0.01$, ${ }^{\star \star *} p<0.001$ compared to group without EMF.
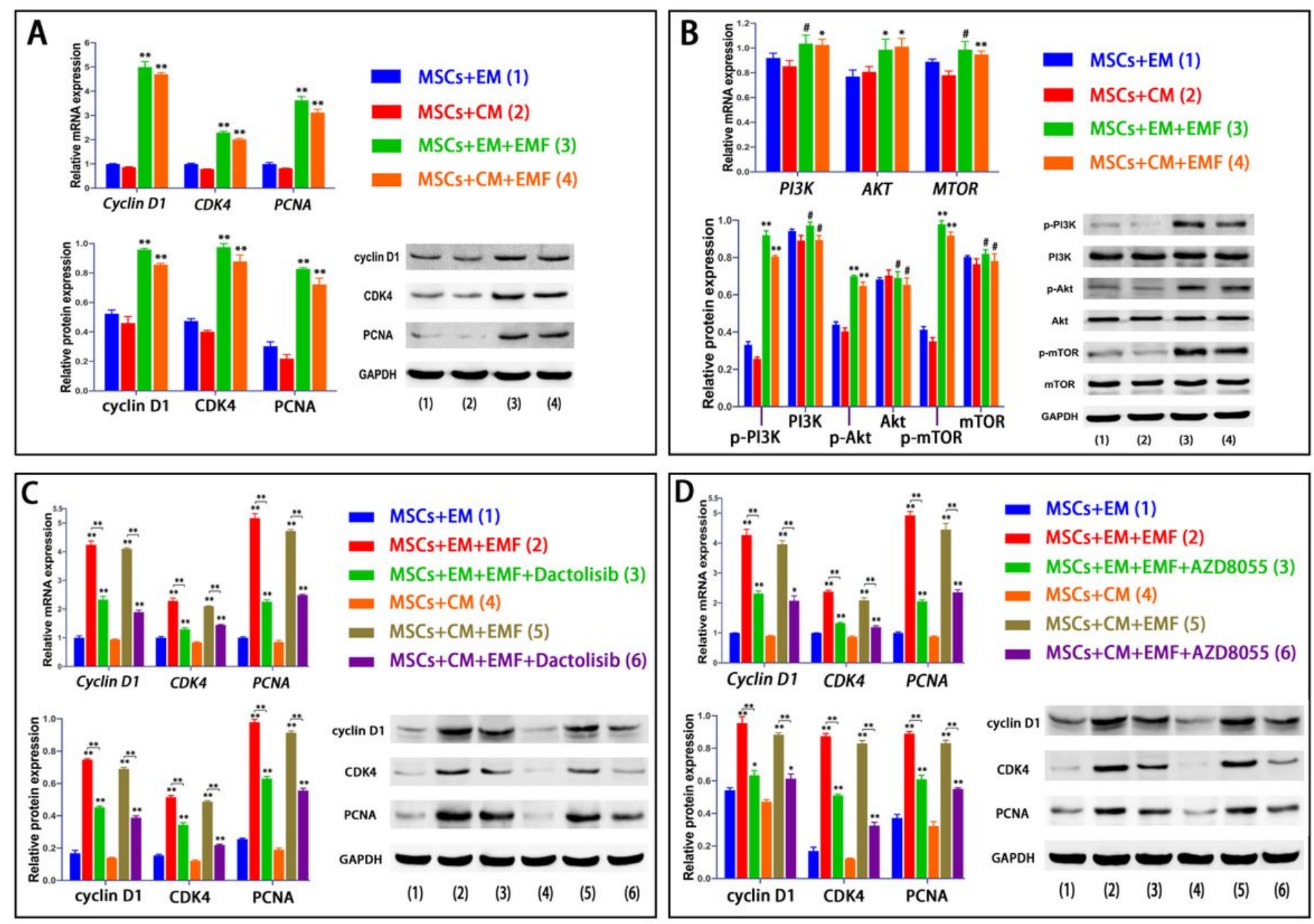

Figure 3

EMF enhanced expressions of proliferation-related genes and proteins partly by activating $\mathrm{PI3K} / \mathrm{Akt} / \mathrm{mTOR}$ pathway. (A) Expressions of Cyclin D1, CDK4, and PCNA were significantly increased in the presence of EMF. (B) Results of qRT-PCR and western blot showed that EMF promoted phosphorylation of PI3K, Akt, and mTOR proteins. (C) PI3K inhibitor (Dactolisib) could significantly reduce the increased expressions of Cyclin D1, CDK4, and PCNA induced by EMF both in gene and protein levels. (D) Inhibition of mTOR by AZD8055 showed results similar to those in (C). Data are shown as mean \pm standard deviation $(n=3)$. \# indicates no significant difference, ${ }^{*} p<0.05,{ }^{* \star} p<0.01,{ }^{* \star} p<0.001$ compared to the group with or without EMF as indicated. 
(1)
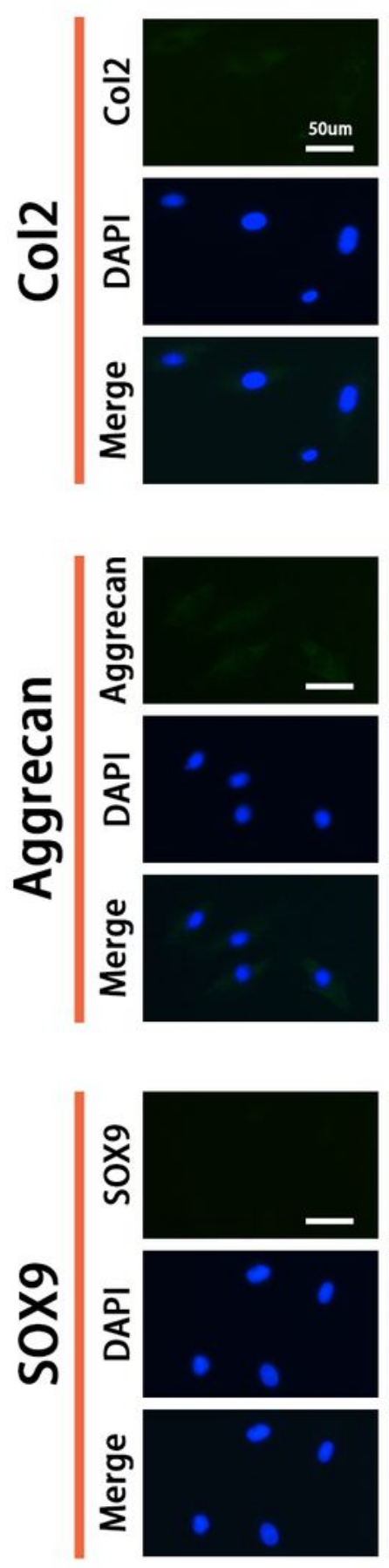

(2)
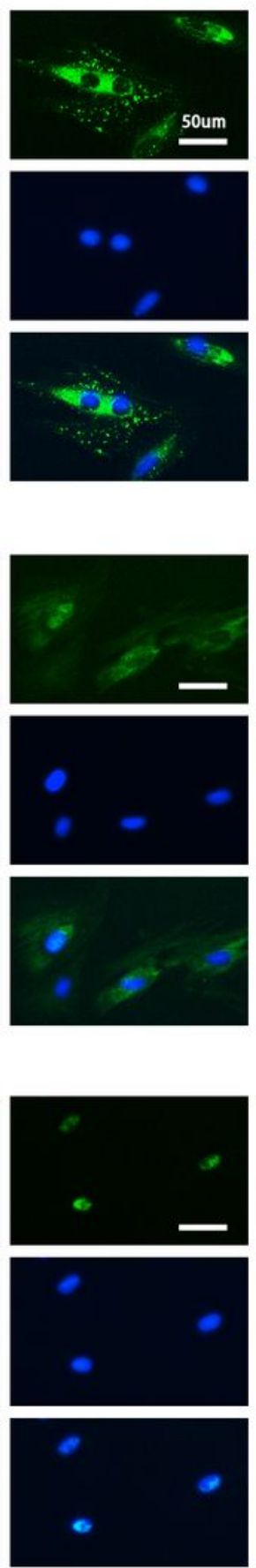

(3)
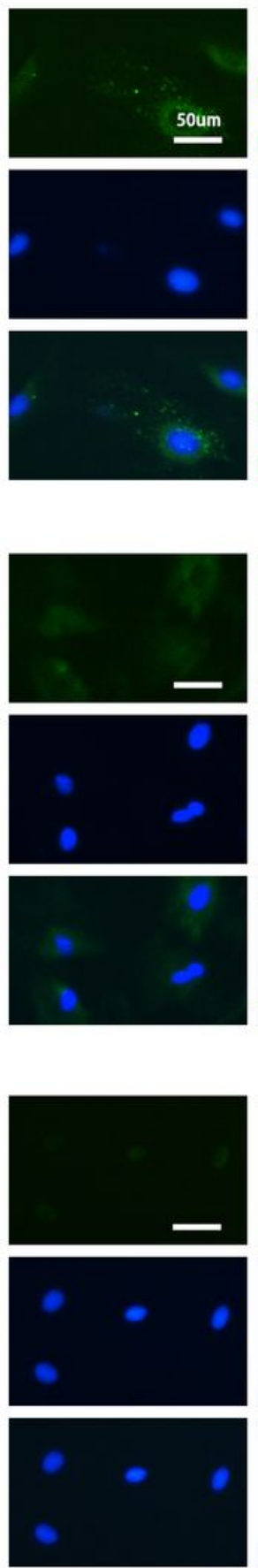

(4)
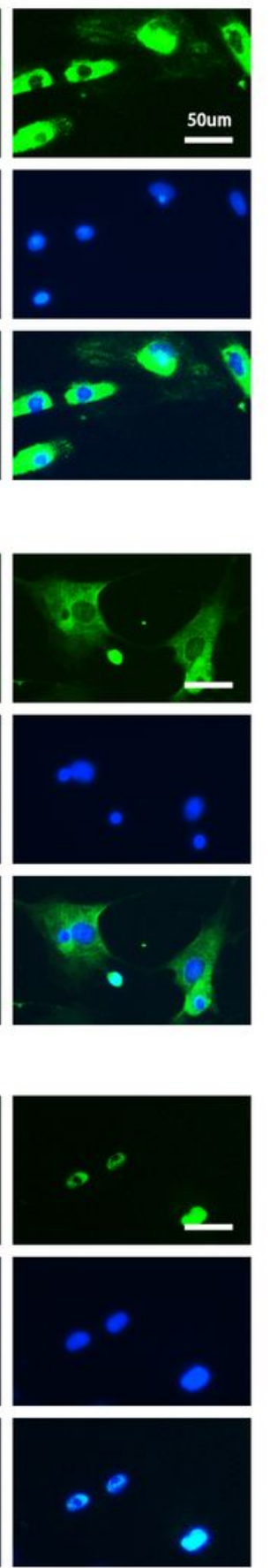
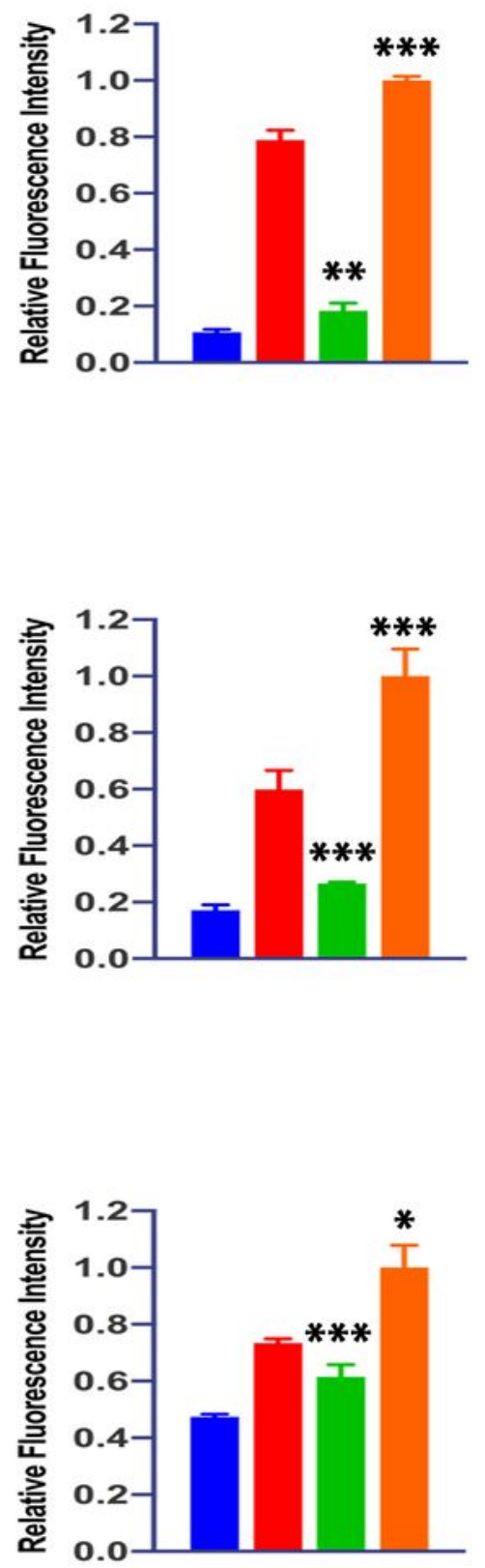

Figure 4

Immunofluorescence analysis of chondrogenesis-associated proteins. Immunofluorescence labeling and quantitative analysis indicate that chondrogenic medium dramatically improved expressions of chondrogenesis-associated proteins (including Col2, Aggrecan, and SOX9) and EMF could further enhance their intensities. Data are shown as mean \pm standard deviation $(n=5)$. \# indicates no significant difference, ${ }^{*} p<0.05,{ }^{* *} p<0.01,{ }^{* *} \mathrm{p}<0.001$ compared to group without EMF. 

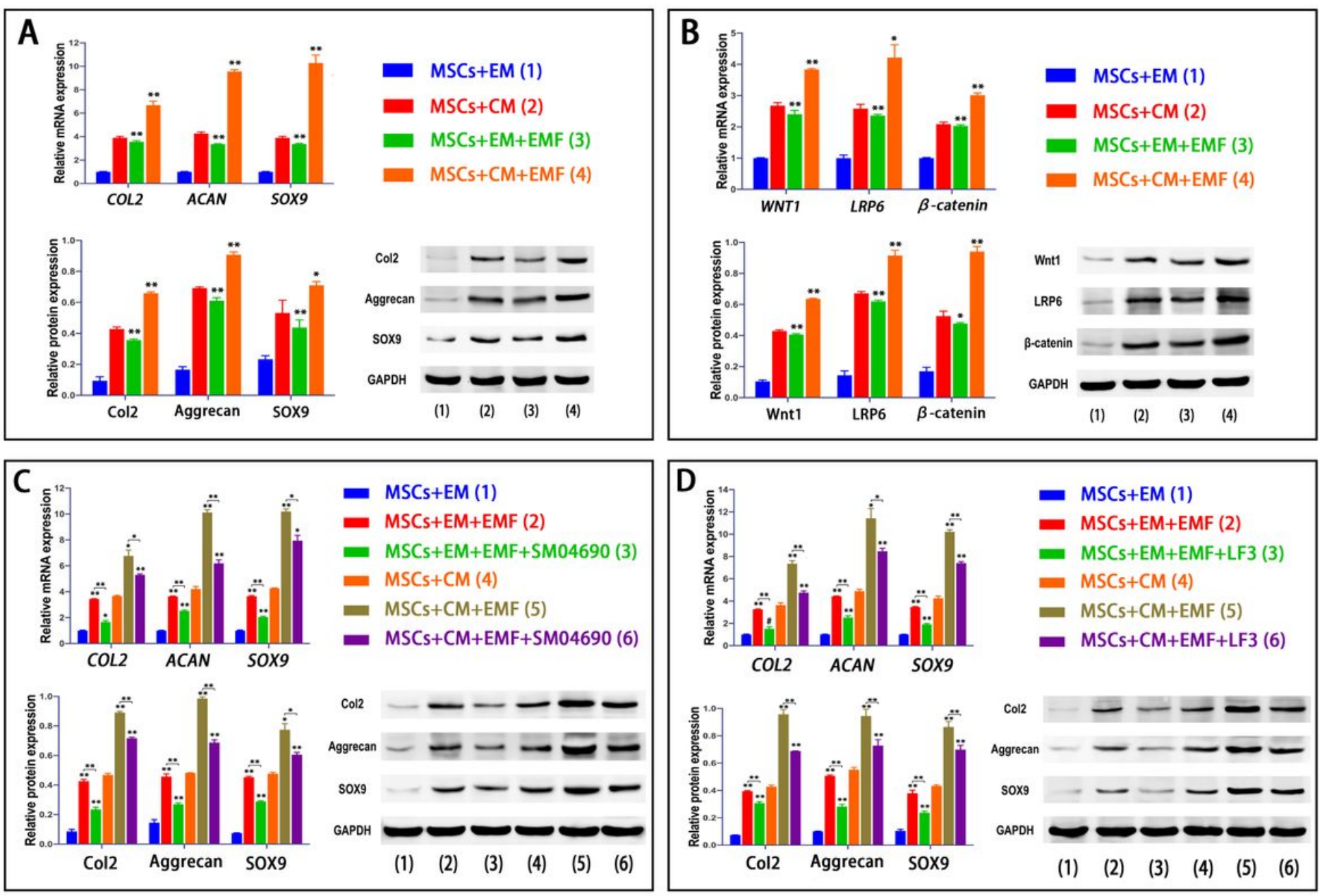

\section{Figure 5}

EMF enhanced chondrogenesis partly by activating Wnt1/LRP6/ $\beta$-catenin pathway. (A) Expressions of Col2, Aggrecan, and SOX9 were significantly increased in the presence of EMF. (B) Results of qRT-PCR and western blot show that EMF activates the Wnt1/LRP6/ $\beta$-catenin signaling pathway. (C) Wnt1 inhibitor (SM04960) significantly reduced the increased expressions of Col2, Aggrecan, and SOX9 induced by EMF both in gene and protein levels. (D) Inhibition of $\beta$-catenin by LF3 shows results similar to those in $(C)$. Data are shown as mean \pm standard deviation $(n=3)$. \# indicates no significant difference, ${ }^{\star} p<0.05,{ }^{\star \star} p<0.01,{ }^{\star \star *} p<0.001$ compared to group without EMF or the group as indicated. 

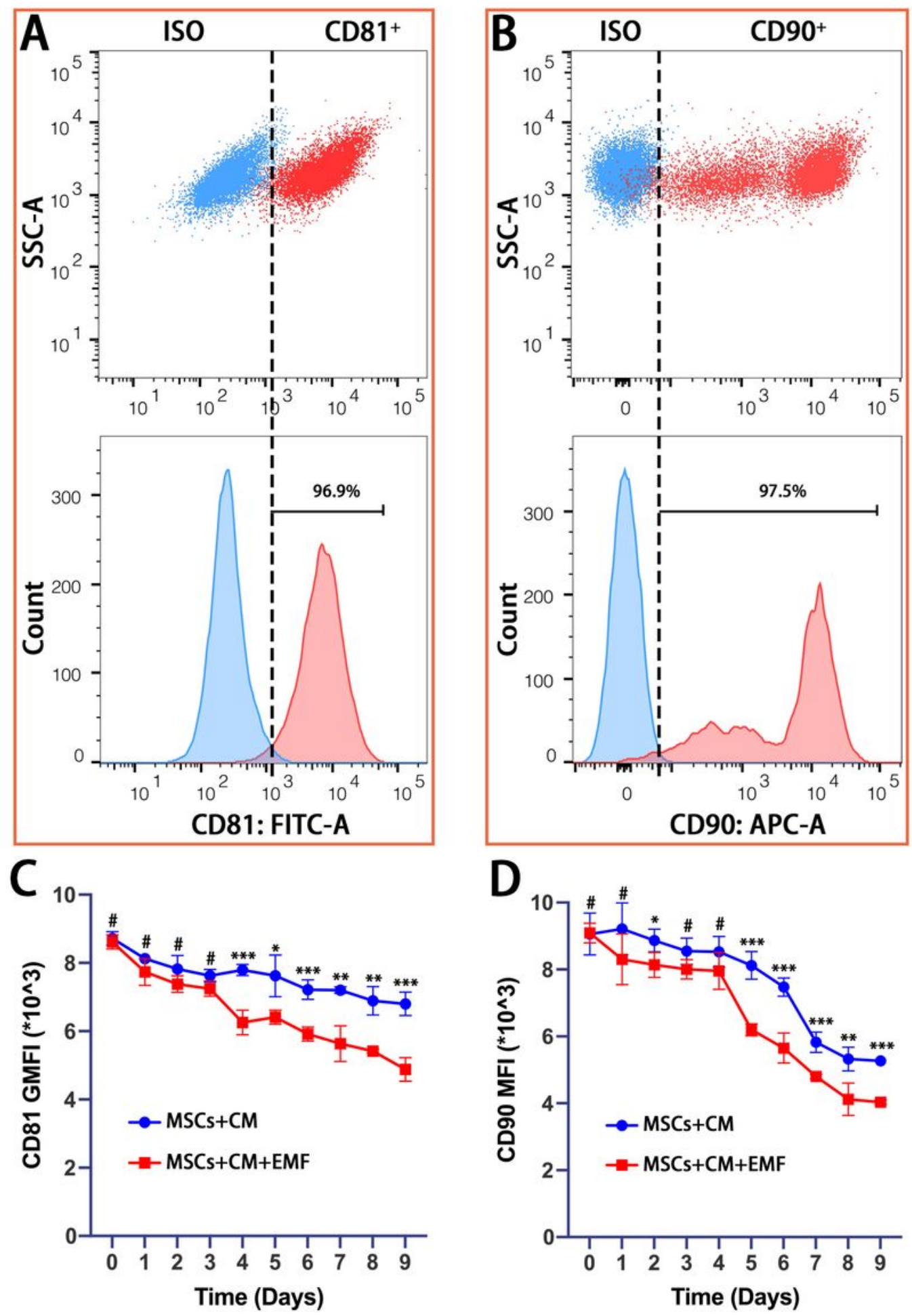

\section{Figure 6}

Identification of BMSCs from rabbits based on two surface markers (CD81 and CD90) and their changes for average fluorescence intensity with time by flow cytometry analysis. (A, B) Results show that $96.9 \%$ and $97.5 \%$ of cells positively express CD81 and CD90, respectively. (C, D) In the culture with chondrogenic medium, the fluorescence intensities of CD81 and CD90 are remarkably decreased in the presence of EMF 
for four and five days, respectively. Data are shown as mean \pm standard deviation $(n=5)$. \# indicates no significant difference, ${ }^{*} p<0.05,{ }^{* \star} p<0.01,{ }^{* \star *} p<0.001$ compared to group without EMF.
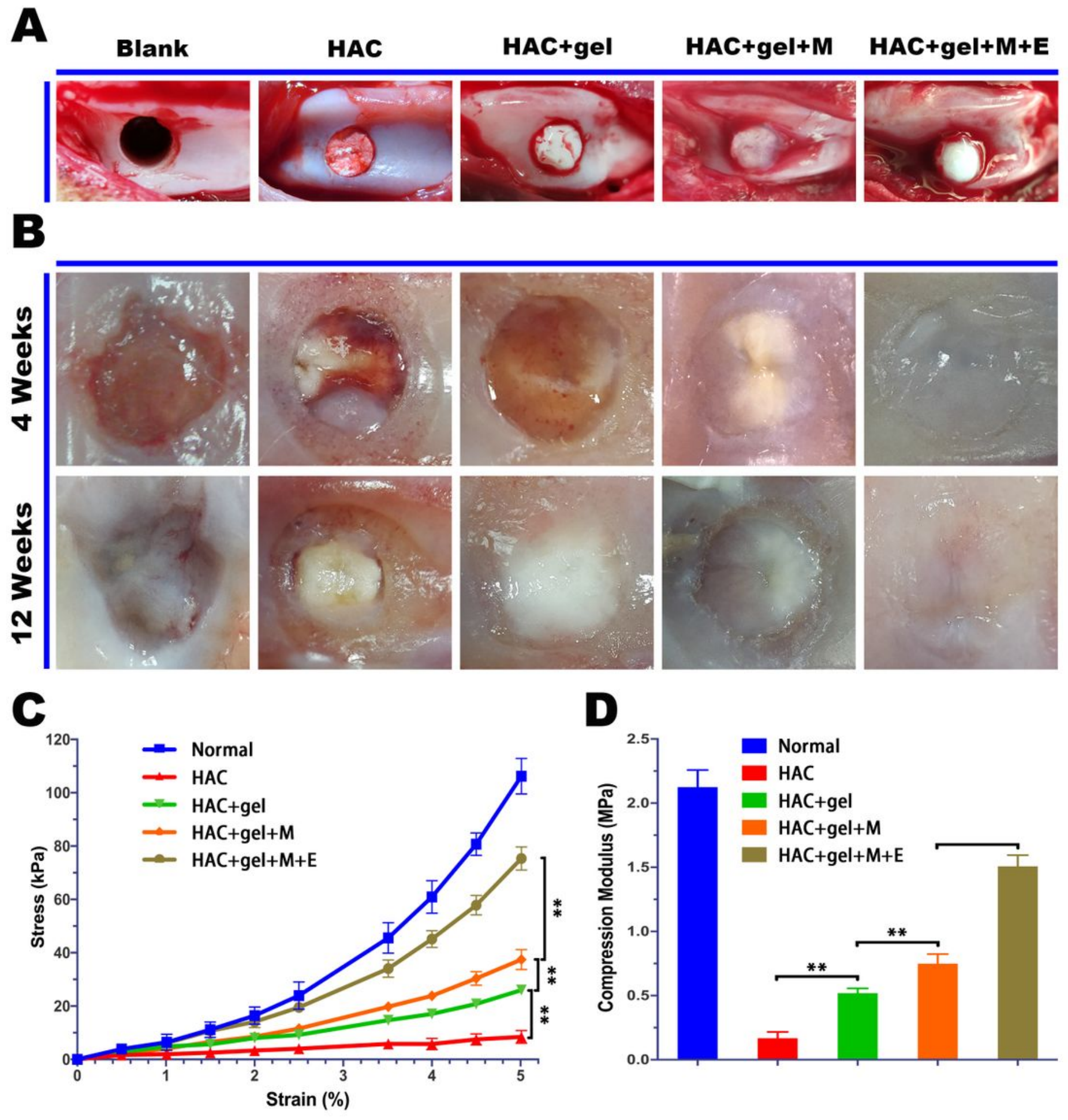

Figure 7

Morphological and mechanical evaluation of regenerative cartilage . (A) Representations of osteochondral defects with different treatments. (B) Macroscopic observation show that osteochondral repair was improved with time from 4 to 12 weeks post implantation. Particularly, the smooth 
neocartilage with similar appearance to adjacent native tissue was found in EMF group. (C) Strain-stress curve of regenerative tissue with representative points at intervals of $0.5 \%$ strain. (D) The compression modulus was calculated at strain of $5 \%$ and the highest modulus in EMF group suggested excellent mechanical properties. Data are shown as mean \pm standard deviation $(n=5)$. \# indicates no significant difference, ${ }^{*} p<0.05,{ }^{*} p<0.01,{ }^{* *} p<0.001$ compared to the group as indicated.

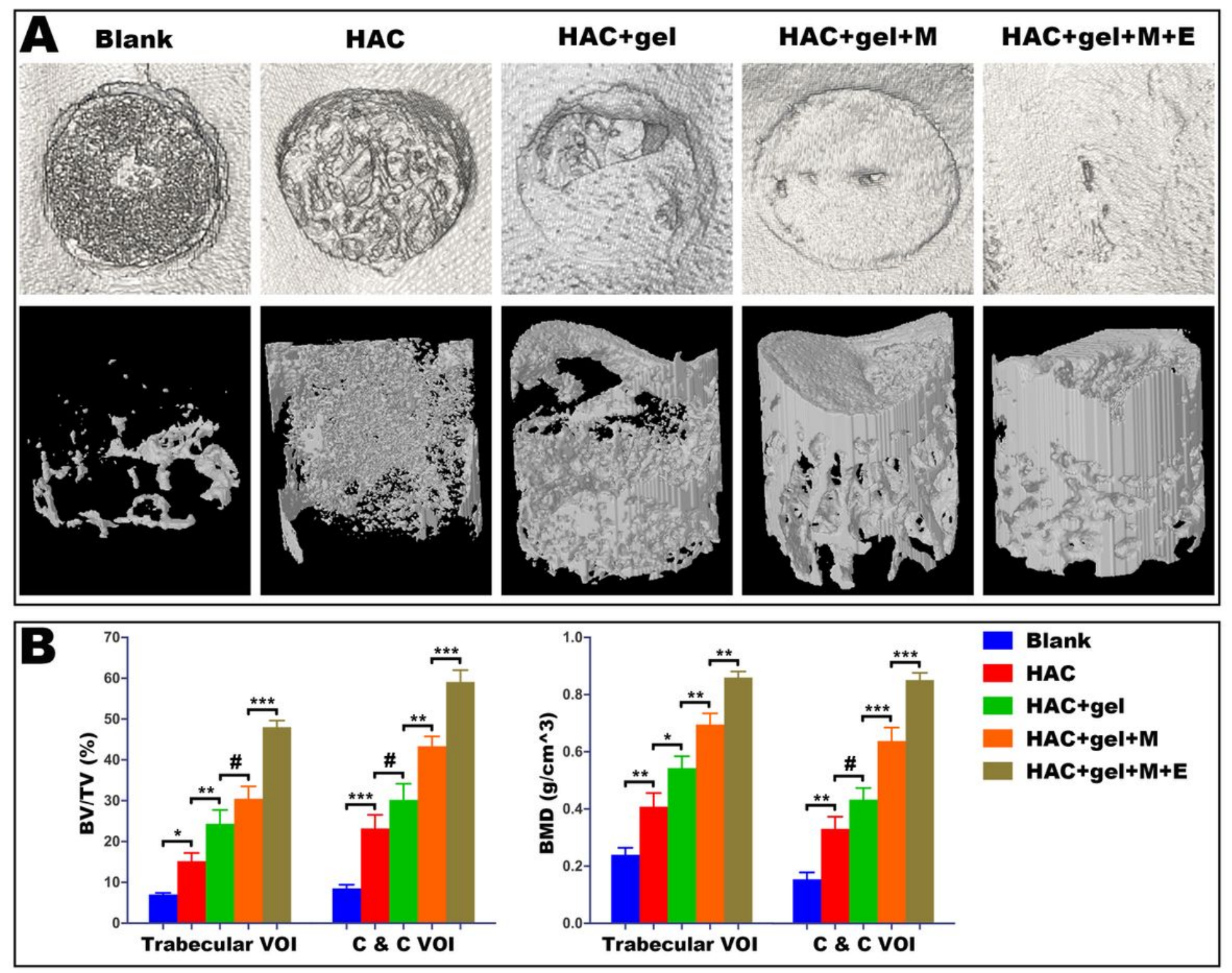

\section{Figure 8}

Micro-CT analysis for subchondral bone regeneration. (A) Reconstructed images of surfaces (first line) and defect area (second line) show improved repair incrementally in groups from left to right. (B) Quantitative analysis of micro-CT data further confirm that bone volume per total volume (BV/TV) and bone mineral density (BMD) were both incrementally increased in groups from left to right, in terms of trabecular volume of interest (VOI) and cartilage and cortical (C\&C) VOI. The values of BV/TV and BMD were highest in the EMF group. Data are shown as mean \pm standard deviation $(n=5)$. \# indicates no significant difference, ${ }^{*} p<0.05, * \star p<0.01,{ }^{* \star} p<0.001$ compared to the group as indicated. 

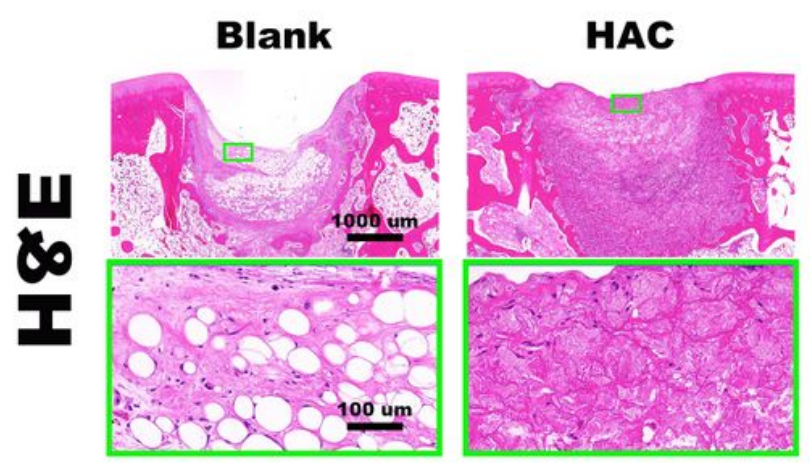

HAC+gel
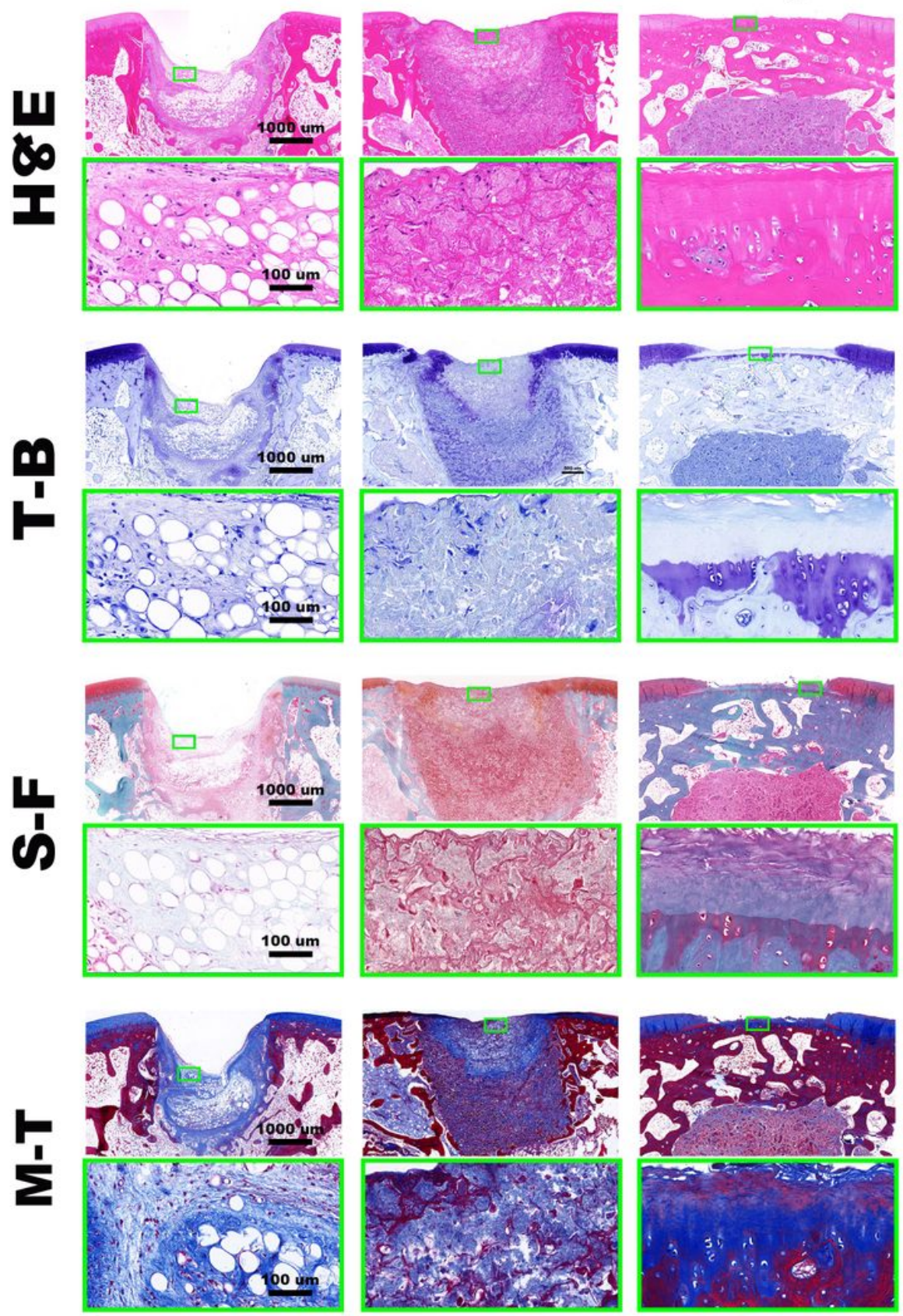
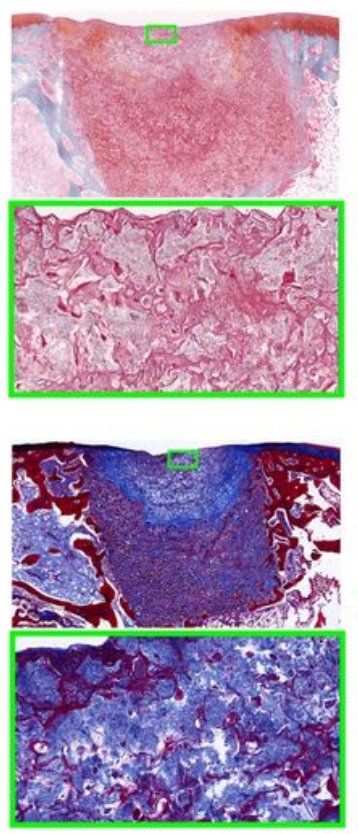
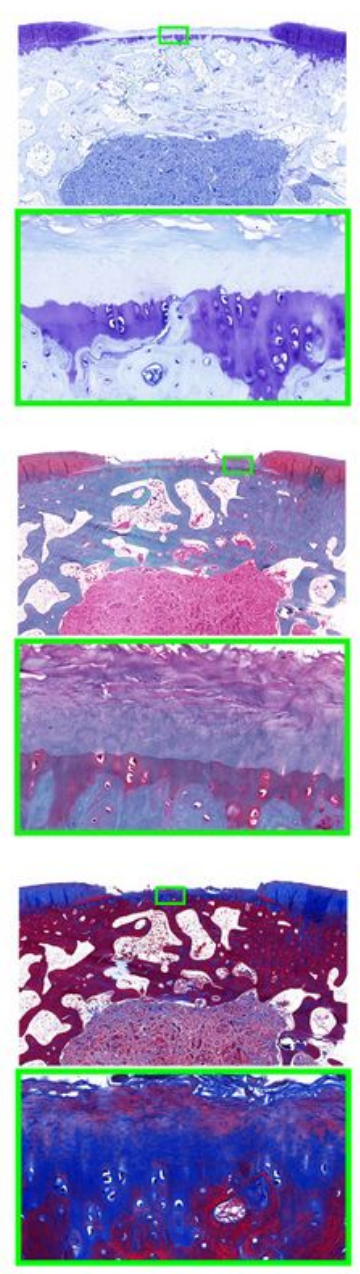

HAC+gel+M
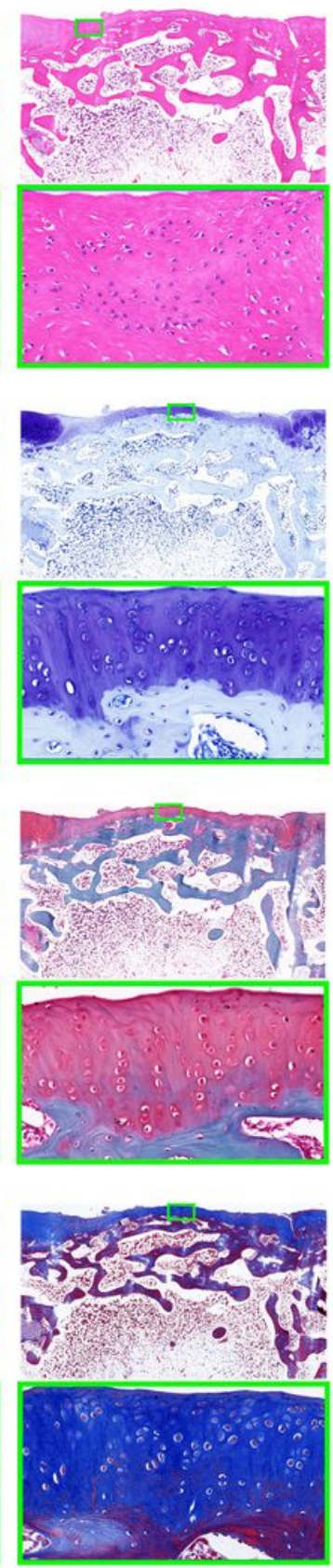

HAC+gel+M+E
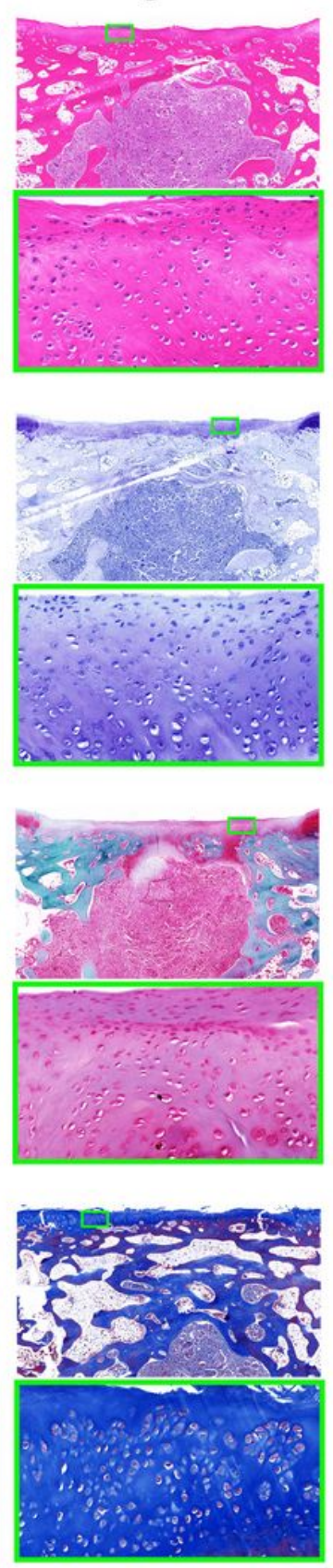

Figure 9

Histological analysis of newly formed tissue at 12 weeks post-surgery. The staining of hematoxylin and eosin (H\&E), toluidine blue (T-B), and Safranin O-fast green (S-F) showed that there was obvious defect in the Blank group, little neocartilage in cell-free groups, and evident repair in cell-loaded groups. In particular, the EMF group had the thickest newly formed tissue in the cartilage layer with most chondrocytes and a well-integrated interface. Masson's Trichrome (M-T) staining also suggested enhanced subchondral bone regeneration and HAC scaffold degeneration in EMF group compared to other groups. 

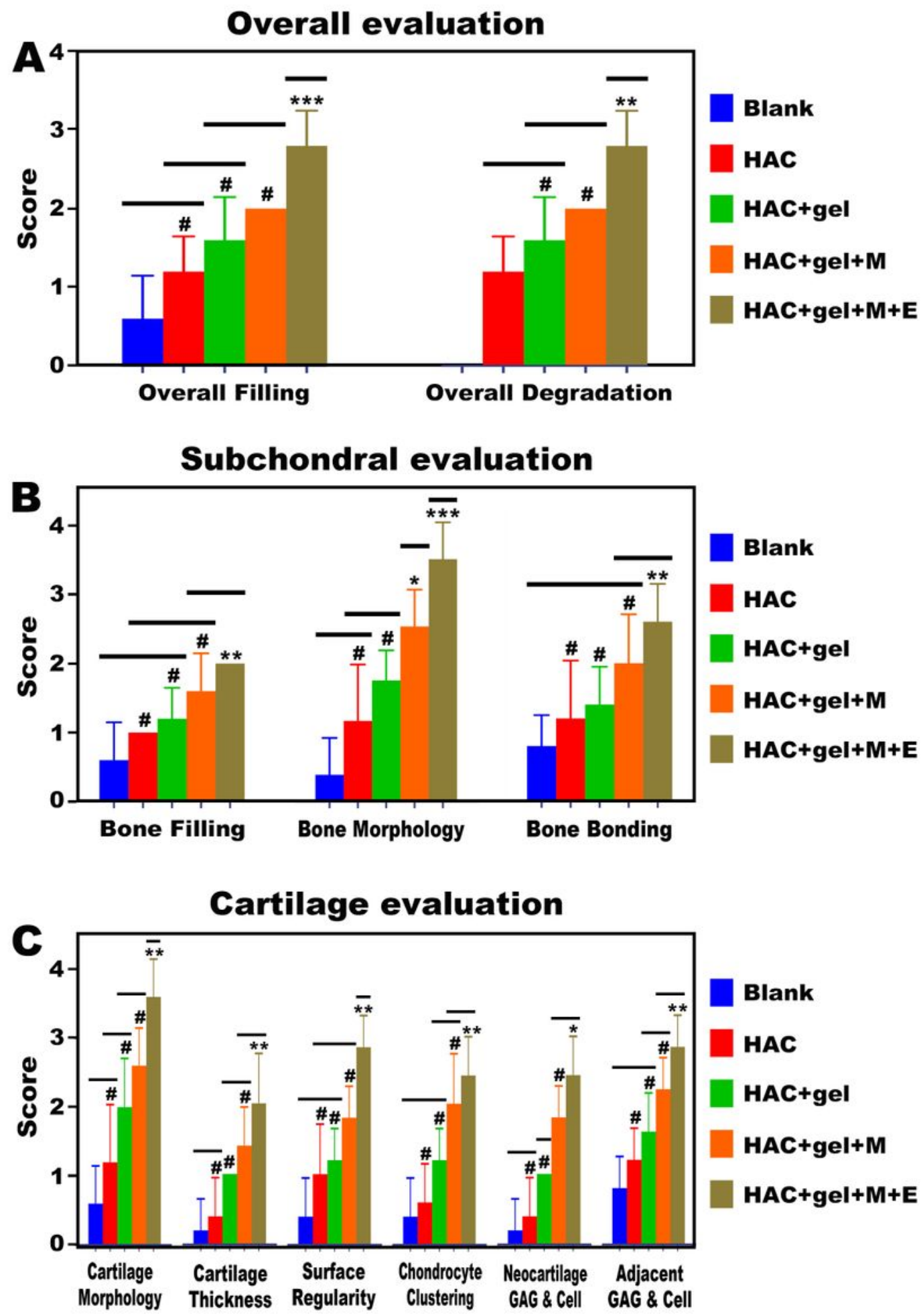

Figure 10

Histological scores for evaluation of osteochondral repair. (A) Scores for overall defect evaluation. (B) Scores for subchondral bone evaluation. (C) Scores for cartilage evaluation. Compared to the Blank group, scores for all parameters except bone morphology were significantly higher in thee EMF group. Data are shown as median (inter quartile range $(I Q R))(n=5)$. \# indicates no significant difference, ${ }^{*} p<$ 
$0.05,{ }^{*} \mathrm{p}<0.01, * \star * \mathrm{p}<0.001$ compared to blank group. The bold horizontal indicates no significant difference among groups under its coverage.

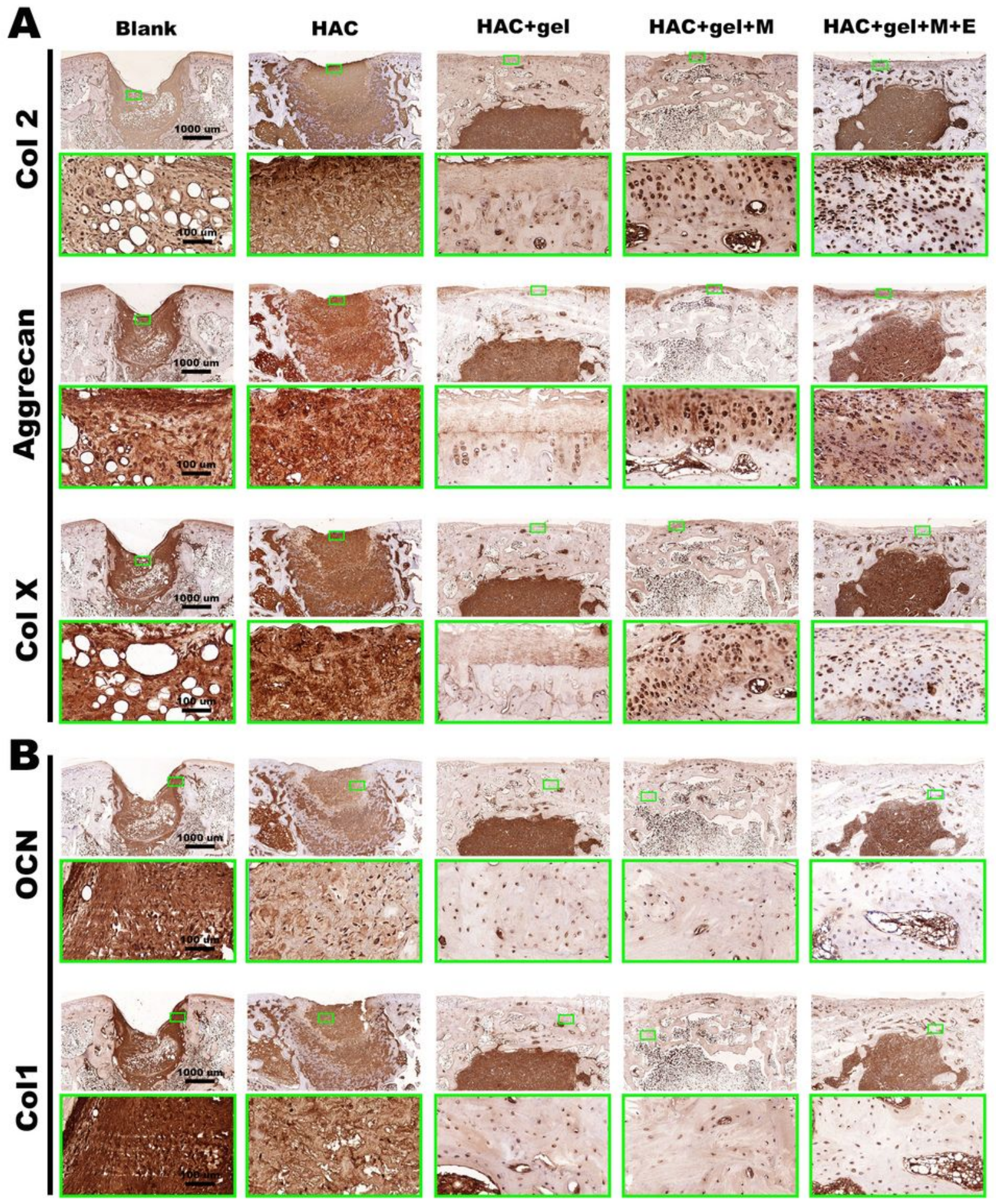

\section{Figure 11}

Immunohistochemical staining for evaluation of chondrogenesis- and osteogenesis-related proteins. (A) Positive staining of Col2, Aggrecan, and Col X was evident in cell-loaded groups, while there were almost no positive cells in the first three groups without BMSCs. The EMF group showed the strongest staining 
of Col2 and Aggrecan proteins. The Col X expression in EMF group was relatively lower compared to the "HAC+gel+M" group. (B) Osteocalcin (OCN) and Col1 were visualized in the last three groups, and their staining was remarkably stronger compared to other two groups. The number of cells positively expressing $\mathrm{OCN}$ or Col1 was relatively higher in the EMF group than those in "HAC+gel" and "HAC+gel+M" groups.

\section{Supplementary Files}

This is a list of supplementary files associated with this preprint. Click to download.

- TableS1.docx

- Tables2.doc

- TableS3.doc 